\title{
tRNA-derived fragments from wheat are potentially involved in susceptibility to Fusarium head blight
}

\author{
Zhengxi Sun ${ }^{\dagger}, Y_{i} \mathrm{Hu}^{\dagger}$, Yilei Zhou, Ning Jiang, Sijia Hu, Lei Li and Tao Li
}

\begin{abstract}
Background: Fusarium head blight (FHB) caused by Fusarium graminearum is a devastating fungal disease of wheat. The mechanism underlying F. graminearum-wheat interaction remains largely unknown. RRNA-derived fragments (tRFs) are RNase-dependent small RNAs derived from tRNAs, and they have not been reported in wheat yet, and whether tRFs are involved in wheat-F. graminearum interactions remains unknown.

Results: Herein, small RNAs from the spikelets inoculated with F. graminearum and mock from an FHB-susceptible variety Chinese Spring (CS) and an FHB-resistant variety Sumai3 (SM) were sequenced respectively. A total of 1249 putative tRFs were identified, in which 15 tRFs was CS-specific and 12 SM-specific. Compared with mock inoculation, 39 tRFs were significantly up-regulated across both wheat varieties after F. graminearum challenge and only nine tRFs were significantly down-regulated. $\mathrm{RRF}^{\mathrm{Glu}}{ }^{\mathrm{A}} \mathrm{tRF}^{\mathrm{Lys}}$ and $\mathrm{RRF}^{\mathrm{Thr}}$ were dramatically induced by F. graminearum infection, with significantly higher fold changes in CS than those in SM. The expression patterns of the three highly induced tRFs were further validated by stem-loop qRT-PCR. The accumulation of tRFs were closely related to ribonucleases T2 family members, which were induced by F. graminearum challenge. The tRFs' targets in host were predicted and were validated by RNA sequencing.
\end{abstract}

Conclusion: Integrative analysis of the differentially expressed tRFs and their candidate targets indicated that tRF Glu, $\mathrm{tRF}^{\mathrm{Lys}}$ and $\mathrm{tRF}^{\mathrm{Thr}}$ might negatively regulate wheat resistance to FHB. Our results unvealed the potential roles of tRFs in wheat-F. graminearum interactions.

Key words: Fusarium head blight, tRNA-derived small RNA fragments, RNase T2

\section{Background}

Fusarium head blight (FHB), mainly caused by Fusarium species complex $[1,2]$, is one of the most devastating fungal disease of wheat. FHB epidemics can cause tremendous yield losses, and also have negative impacts on human and animal health due to mycotoxins

\footnotetext{
*Correspondence: taoli@yzu.edu.cn

${ }^{\dagger}$ Zhengxi Sun and Yi Hu contributed equally to this work.

Key Laboratory of Plant Functional Genomics of the Ministry

of Education/Jiangsu, Key Laboratory of Crop Genomics and Molecular

Breeding/Collaborative Innovation of Modern Crops and Food Crops

in Jiangsu/Jiangsu Key Laboratory of Crop Genetics and Physiology,

College of Agriculture, Yangzhou University, Yangzhou 225009, China
}

deoxynivalenol (DON) contamination [3]. DON negatively regulates the protein synthesis by inhibiting the function of ribosome [4], and it also leads to cellular toxicity by inhibition of DNA and RNA synthesis [5], alteration of membrane structure [6], and by suppression of mitochondrial function and cell-cycle $[7,8]$.

Large number of quantitative trait loci (QTL) associated with FHB-resistance have been reported, and only two QTL (Fhb1 and Fhb7) have been claimed to be cloned, however, their functions remain controversial [9-13], and our knowledge on the mechanism underlying wheat-F. graminearum interaction is still quite limited. 
Transfer RNAs (tRNAs) are essential components of the translation machinery, and they also play roles in modulating cell proliferation and stress responses [14, 15]. tRFs can derive from tRNA precursor, but most are generated from cleavage of mature tRNAs [16, 17]. tRFs were classified into three types according to the region of cleavage and their size: long tRFs (circa 30-35 nt) originating from tRNA cleavage in the anticodon region, short tRFs (<28 nt) from cleavage either in the $\mathrm{D}$ or $\mathrm{T}$ region [18], and tiny tRFs (10-16 nt) [19]. Angiogenin, a kind of RNase A, is involved in the biogenesis of the long tRFs in human [20]. In yeast and protozoan, the long tRFs were produced through cleavage of tRNAs by Rny1p and Rnt2 respectively [21, 22]. Concerning short tRFs, DICER 1 or Dicer-like protein 1 (DCL1) was shown to cleave the $\mathrm{D}$ or $\mathrm{T}$ region of some tRNAs [17, 23-25]. However, RNases T2, instead of Dicer protein, was proposed to be involved in plant $\mathrm{tRF}$ biosynthesis [26]. The sizes of tiny tRFs range from 13 nt to 16 nt in Arabidopsis [19]. tRFs resemble the functional characteristics as microRNAs, including binding to AGO proteins, RISC complex formation with AGO proteins, and RNA silencing [24, 27-31]. The previous reports indicated that tRNAs were endonucleolytically cleaved under a variety of stress conditions [32]. tRFs were found in malignant human tumors and most of of them played pivotal functions in cancer progression and metabolic diseases [33-35]. tRFs firstly described in Escherichia coli responded to bacteriophage infection [36]. Recent report showed that RNase T2 was involved in the response to pathogen challenges [37]. Rhizobial tRNA-derived small RNAs were involved in cross-kingdom regulation of soybean nodulation [38]. tRFs in land plants were reported to participate in abiotic stress and development $[19,39,40]$. Until now, the databases about tRF in several plant species have been established, such as Arabidopsis, rice, soybean, maize, and grape (http://14.139.61.8/PtRFdb/index.php) [19, 41]. Wheat is one of the most important staple crops in the world, however, our knowledge on wheat tRFs is quite limited and the responses of wheat tRFs to $F$. graminearum challenge have not been reported yet.

In this study, through small RNA sequencing, tRFs in wheat under $F$. graminearum challenge and mock inoculation were examined; sizes and abundances of tRFs were analyzed; tRFs responsive to F. graminearum infection, their targets in host, and their potential functions in wheat- $F$. graminearum interaction were addressed. To our knowledge, this is the first report on tRFs in wheat responses to FHB. We hope the outcome of this study may provide a novel insight into the interactions between wheat and F. graminearum. Meanwhile, our data serves as a useful resource of wheat tRFs for further studying.

\section{Results}

Identification of tRFs in wheat spikelet by small RNA-seq

Different responses to F. graminearum infection between CS and SM were observed, and only one or two symptomatic spikelets appeared on SM at 10 days post inoculation (dpi), whereas four or five symptomatic spikelets on CS (Fig. S1). Twelve libraries constructed from the total RNAs of the spikelets of CS and SM with F. graminearum infection and mock inoculation, respectively, were subjected to deep sequencing. Approximately 27 million raw tags of small RNA were generated in each library. At least 22 million clean tags in each library were mapped to the wheat genome. The Q20 of the clean tags were up to 99\% (Table S1.1). The expressions of siRNAs were significantly downregulated by $F$. graminearum infection when compared with mock inoculation in both varieties, however, the expressional abundance of siRNAs in SM were significantly higher than their counterparts in CS across mock and F. graminearum inoculations (Fig. 1a). The number of tRFs was more than that of miRNAs under both $F$. graminearum and mock inoculations across two varieties, and the lengths predominantly ranged from 18 to 20 nt (Fig. 1a, b). Totally 1249 putative tRFs were identified, and all were derived from the tRNAs for transferring 20 types of amino acids, and the majority of them derived from the $5^{\prime}$ end of the tRNAs (Table S2, Fig. S2). A total of $53,110,35$ and 59 of specific tRFs were detected in CSM, CSI, SMM and SMI group respectively (Fig. 1c, Table S3), and 568 tRFs were in common across the twelve libraries, and 147 out of the $568 \mathrm{tRFs}$ were enriched for at least 500 transcripts per million reads (TPM) in any of the four contrasting groups (Fig. 1c, Table S4). The heat map for the 147 tRFs clearly showed that tRF ${ }^{\mathrm{Glu}(\mathrm{CUC})}, \mathrm{tRF}^{\mathrm{Lys}(\mathrm{CUU})}$ and $\mathrm{tRF}^{\mathrm{Th}(\mathrm{CGU})}$ ranked top 3 in abundance both in CS and SM (Fig. 1d).

\section{Wheat tRFs were accumulated after infection by $F$. graminearum especially in FHB-susceptible variety}

Responsive patterns of tRFs to $F$. graminearum and mock inoculation were compared between CSI and CSM, and between SMI and SMM. Seventy-four tRFs had significant differences in abundance between CSM and CSI, with 47 tRFs being upregulated and 27 downregulated after F. graminearum infection (Fig. 2a). Fifty-nine tRFs had significant differences in abundance between SMM and SMI, with 49 tRFs being upregulated and 10 downregulated after $F$. graminearum infection (Fig. 2b). We also compared the expressional patterns of tRFs between the two varieties, SMM versus CSM, and SMI versus CSI. CS accumulated more tRFs across the two contrasting inoculations (Fig. 2c, d). It is surprising that all of the significantly differentially expressed $(p<0.05)$ tRFs appeared only in CS (Fig. 2c, 
d). Forty-eight significantly differentially expressed tRFs were associated with $F$. graminearum infection, with $\mathrm{tRF}^{\mathrm{G} \ln (\mathrm{UUG})}$ and $\mathrm{tRF}^{\mathrm{Val}(\mathrm{CAC})}$ showing largest changes (Fig. 2e, f). Interestingly, 14 of the 48 tRFs were derived from tRNA-Lys (Table 1). Moreover, all of the $14 \mathrm{tRF}^{\mathrm{Lys}}$ were dramatically accumulated by $F$. graminearum infection both in CS and SM, but the abundances in CS were higher than those in SM. tRFs derived from tRNA-Lys-CUU-6-1 were CS-specific (Table S3.5). Interestingly, one tRNA, tRNA-Lys-CUU-10-1, produced diversiform short fragments. The tRFs primarily derived from the $5^{\prime}$ end of the tRNAs, and only five tRFs derived from the $3^{\prime}$ end of the tRNAs (Table S5, Fig. S2). The majority of the tRFs varied from 18 to 21 nt in length, and only a few tRFs had size up to $25 \mathrm{nt}$, such as $\mathrm{tRF}^{\mathrm{Ala}(\mathrm{AGC})}$ and $\mathrm{tRF}^{\mathrm{Cys}(\mathrm{GCA})}$ (Fig. S3).

Clone sequencing of the top three tRFs in abundance, $t_{R} F^{\mathrm{Glu}(\mathrm{CUC})}, \mathrm{tRF}^{\mathrm{Lys}(\mathrm{CUU})}$ and $\mathrm{tRF}^{\mathrm{Thr}(\mathrm{CGU})}$, validated the tRFs from small RNA sequencing (Supplemental Data 1 ). The primary structures of tRNA indicated that all the three tRFs derived from the 5' end of tRNAs (Fig. 3a, b, c). Stem-loop qRT-PCR validated the differentially expressed tRFs from small RNA-Seq, and these three tRFs were dramatically induced by $F$. graminearum in both varieties, but CS accumulated significantly more tRFs than those in SM (Fig. 3d, e, f). At same time, we checked the expressions of these three tRFs at different time points (Fig. S4). tRF ${ }^{\text {Glu }}$ was induced at 2 days post F. graminearum inoculation (dpi) in CS, but at $3 \mathrm{dpi}$ in $\mathrm{SM}$, and the degree of induction in CS was significantly higher than that in SM at 6 dpi (Fig. S4a). tRF ${ }^{\mathrm{Lys}}$ was significantly induced at $2 \mathrm{dpi}$ and $5 \mathrm{dpi}$ in CS, whereas was slightly induced at $5 \mathrm{dpi}$ in SM (Fig. S4b). tRF ${ }^{\text {Thr }}$ was induced at $12 \mathrm{~h}$ after inoculation with $F$. graminearum in both varieties, but was strongly induced at 2 dpi in SM. At later stages of F. graminearum infection, tRF was significantly induced only in CS (Fig. S4c). In short, the expression patterns of all these three tRFs reflect a common rule, that is, they were induced in CS, and the induction amplitude gradually increased with the temporal infection progression.

\section{RNase T2 was closely associated with the formation of tRFs}

To understand the association of RNase T2 with tRF formation and accumulation, the changes of wheat RNase T2 family members were analyzed under $F$. graminearum and mock inoculations. S-like RNase genes RNS1,
RNS2, RNS3 and RNS4 are RNase T2 genes in Arabidopsis. Blasting RNS1, RNS2, RNS3 and RNS4 against wheat genome database identified nine wheat RNase $\mathrm{T} 2$ orthologues, which were located in Chrs.2A/2B/2D, Chrs.3A/3B/3D and Chrs.6A/6B/6D, respectively (Fig. 4a). Four of them (TraesCS2B02G182900, TraesCS2D02G163300, TraesCS3A02G398300 and TraesCS3D02G392300) were significantly induced by $F$. graminearum challenge of CS, and their intensities of induction in CS were significantly higher than their counterparts in SM (Fig. S5, Fig. 4b). There were no significant changes or only low expressional levels for the other five RNase T2 genes, suggesting that not all RNase T2 members were induced by $F$ graminearum to cleave the accumulated tRNAs in wheat. Notably, TraesCS3A02G398300 was significantly induced and might be the main producer of tRFs (Fig. 4b). Additionally, we analyzed the expressions of TaAGO1 and TaDCL family members in our transcriptome data. Surprisingly, all of TaAGO1 and TaDCL family members were significantly inhibited by $F$. graminearum across two wheat varieties (Figure S6). These results further illustrated that RNase $\mathrm{T} 2$ probably played a pivotal role in tRF biosynthesis.

\section{Prediction and validation of tRFs' targets in wheat}

Twelve mRNA libraries corresponding to the 12 small RNA libraries were constructed to identify the potential tRFs' targets. An average of 120 million raw reads of mRNA was generated in each library, and at least 103 million clean reads in each library were mapped to the wheat genome. Q20 of the clean reads was up to $96 \%$ (Table S1.2). Up to 100 target genes of high confidence for all the identified tRFs were predicted in wheat (Table S6). Notably, almost all of the predicted targets in CS and SM were downregulated after infection by $F$. graminearum, and the extent of downregulation was stronger in CS than that in SM (Fig. 5a). The expressional levels of the tRFs' targets were negatively associated with the expressions of the tRFs (Table 2), suggesting tRFs might inhibit the expressional level of their targets as miRNAs' action. Gene Ontology (GO) classification showed that the terms "cellular process", "metabolic process" and "response to stimulus" were dominantly enriched in the biological process category. In the cellular component ontology, "cell", "organelle" and "membrane" were the highly abundant categories. The genes dramatically enriched in the molecular function category were involved in "catalytic

(See figure on next page.)

Fig. 1 Summary of tRFs from sRNA-Seq. (a) Total counts of miRNA, siRNA and tRF in different groups. (b) Length distribution of tRFs. Count values are shown as means \pm standard errors (s.e.) over three biological replicates. Different letters indicate significant differences according to the Student-Newman-Keulsa test $(p<0.05)$. (c) Venn distribution of the identified tRFs among the four libraries. (d) Expression and cluster analysis of 147 highly expressed tRFs among the four libraries 
(a)

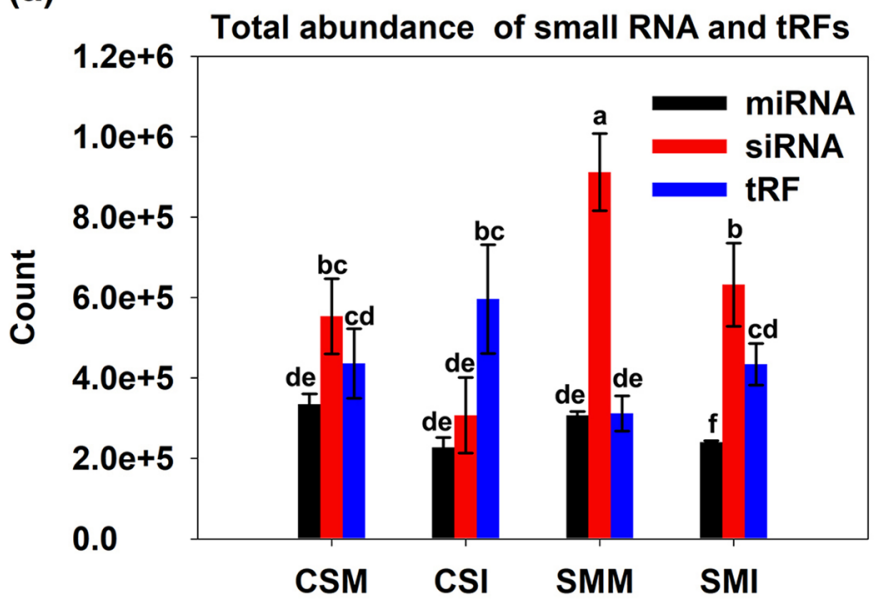

(b)

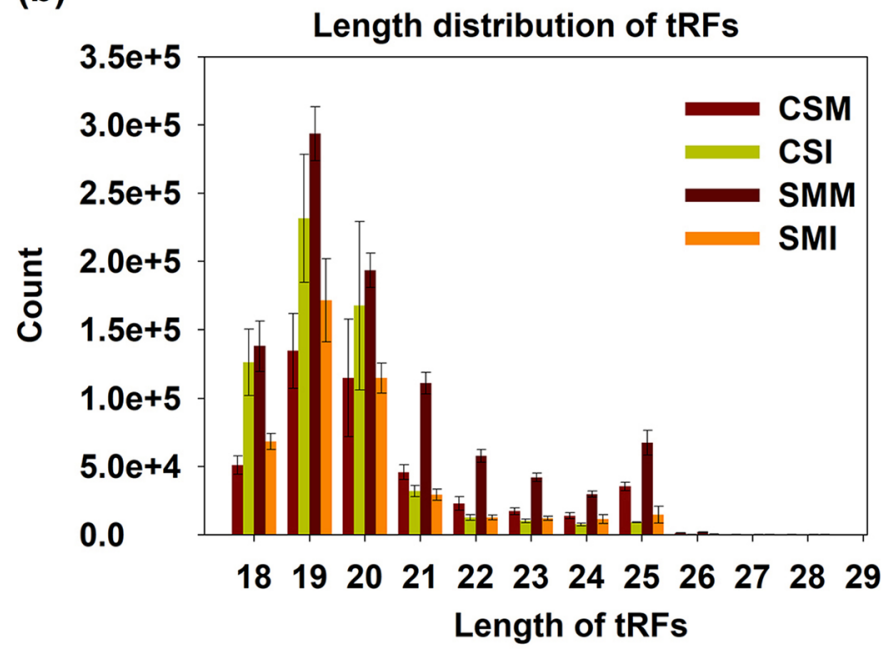

(c)

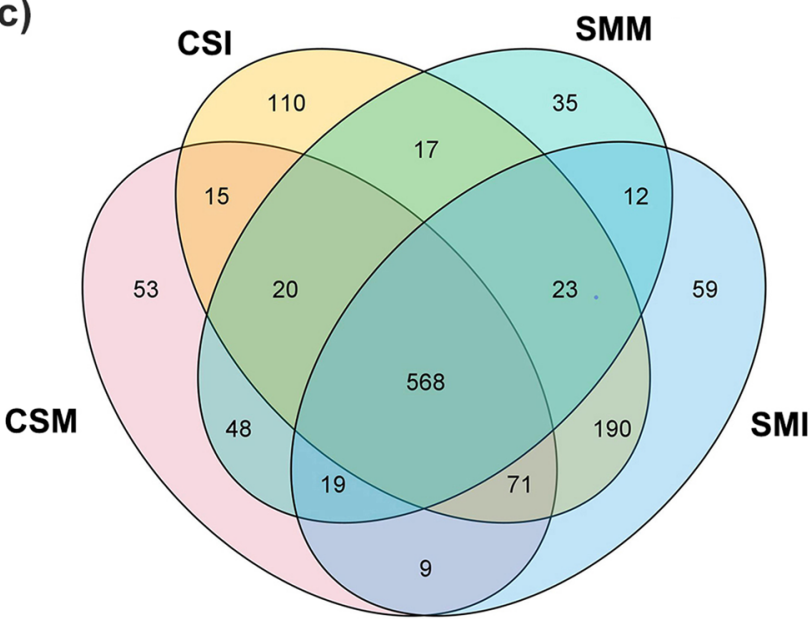

(d)

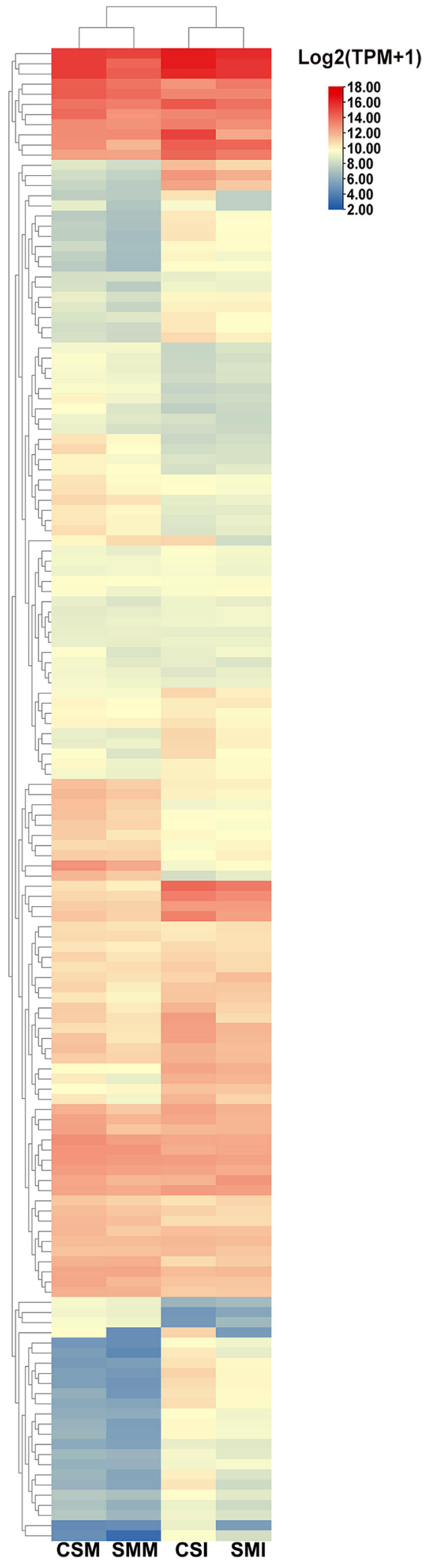




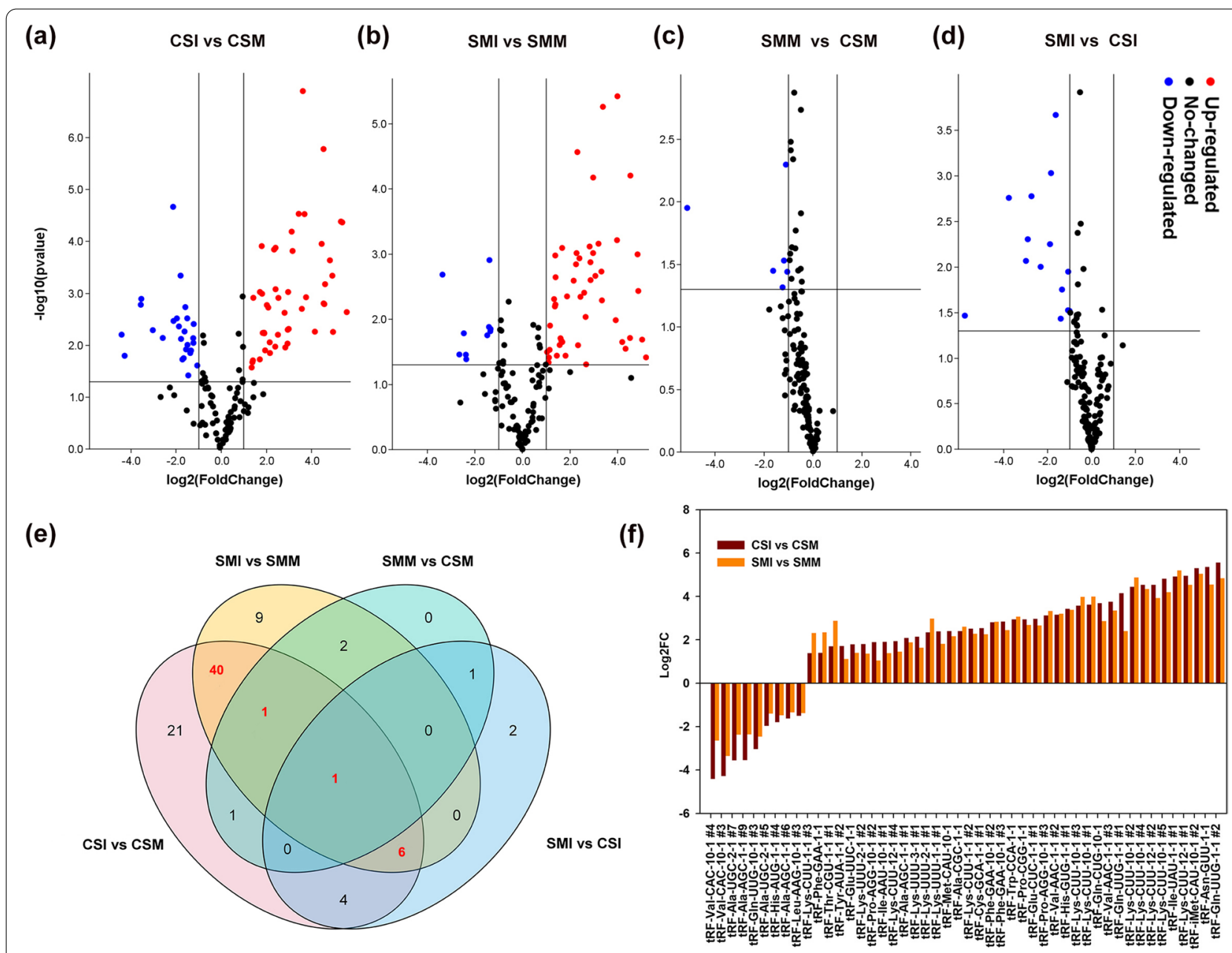

Fig. 2 Differentially expressed wheat tRFs between F. graminearum and mock inoculation of CS and SM. Volcano plots show the comparisons between CSI and CSM (a), SMI and SMM (b), SMM and CSM (c) , SMI and SMM (d). X-axis represents the Log2(fold change). Y-axis represents the $p$ value in biological replication. The plots in red show the up-regulated genes, blue plots show the down-regulated genes, and blank plots show the unchanged genes. Each plot represents one gene. The significant differences were analyzed by $t$ testing with the significance threshold of 0.05 . (e) Venn diagram shows the distribution of differentially expressed tRFs among comparisons of the four libraries. Red in bold represents the number of the tRFs associated with F. graminearum infection. (f) Fold changes of 48 differentially expressed tRFs in comparisons of CSI-vs-CSM and SMI-vs-SMM

activity" and "binding" (Fig. 5b). The most enriched GO terms were those associated with the proteasome components or its organization, followed by galactolipid biosynthetic and metabolic process (Fig. 5d). DNA damage and repair related terms were also significantly enriched. Kyoto Encyclopedia of Gene and Genomes (KEGG) pathway analysis showed "metabolism pathway" was the most represented pathway, including "carbonhydrate metabolism", "biosynthesis of other secondary metabolites", "glycan biosynthesis and metabolism", "lipid metabolism", "amino acid metabolism" and "nucleotide metabolism", and most of these genes were classified into "carbonhydrate metabolism" pathway (Fig. 5c). "ascorbate and aldarate metabolism" and "lysine degradation" were also significantly enriched (Fig. 5e). The KEGG pathway enrichment analysis supported the results from the GO enrichment analysis, such as "proteasome", "glycerolipid metabolism" and "purine metabolism".

In order to capture the key tRFs targets, protein interaction analysis was performed. Four hub tRFs targets were uncovered, TraesCS2B02G598800, TraesCS6D02G254900, TraesCSU02G123400 and TraesCS2A02G141800 (Fig. 6), which were the candidate target genes of $\mathrm{tRF}^{\mathrm{iMet}(\mathrm{CAU})}, \mathrm{tRF}^{\mathrm{Lys}(\mathrm{CUU})}, \mathrm{tRF}^{\mathrm{Ile}(\mathrm{GAU})}$ and $\mathrm{tRF}^{\operatorname{Th}(\mathrm{CGU})}$, respectively (Table S6).

qRT-PCR was performed to validate the tRFs' target genes predicted from RNA-sequencing data. Ten predicted targets of $\mathrm{tRF}^{\mathrm{Lys}(\mathrm{CUU})}$ and $\mathrm{tRF}^{\mathrm{Thr}(\mathrm{CGU})}$ were 
Table 1 Differentially expressed tRFs between CS and SM after F. graminearum (CSI and SMI) or mung bean broth (CSM and SMM) inoculation

\begin{tabular}{|c|c|c|c|c|c|c|c|c|}
\hline tRF ID & Sequence & Length & CSM & CSI & SMM & SMI & $\begin{array}{l}\log 2 \\
\text { (CSI/CSM) }\end{array}$ & $\begin{array}{l}\log 2 \\
\text { (SMI/SMM) }\end{array}$ \\
\hline tRF-Ala-AGC-1-1 \#1 & GCGAGAGGTACGGGGATCG & 19 & 132 & 557 & 73 & 267 & 2.08 & 1.88 \\
\hline tRF-Ala-AGC-1-1 \#6 & GGGGATGTAGCTCAGATGGTAG & 22 & 3470 & 1122 & 2600 & 1020 & -1.63 & -1.35 \\
\hline tRF-Ala-AGC-1-1 \#9 & GGGGATGTAGCTCAGATGGTAGAGC & 25 & 7892 & 676 & 4697 & 914 & -3.54 & -2.36 \\
\hline tRF-Ala-CGC-1-1 & GCGAGAGGCACGGGGTTCG & 19 & 198 & 1048 & 108 & 654 & 2.40 & 2.60 \\
\hline tRF-Ala-UGC-2-1 \#5 & GGGGATGTAGCTCAAATGGTAG & 22 & 1849 & 472 & 1534 & 578 & -1.97 & -1.41 \\
\hline tRF-Ala-UGC-2-1 \#7 & GGGGATGTAGCTCAAATGGTAGAGC & 25 & 3663 & 309 & 2472 & 475 & -3.57 & -2.38 \\
\hline tRF-Asn-GUU-1-1 & AACCACAAGGTCGGAGGT & 18 & 36 & 1493 & 41 & 963 & 5.36 & 4.54 \\
\hline tRF-Cys-GCA-1-1 \#1 & GGGTCCATAGCTCAGTGG & 18 & 1888 & 10909 & 1762 & 8410 & 2.53 & 2.26 \\
\hline tRF-GIn-CUG-10-1 & TCCAGTAACCCGAGTTCA & 18 & 90 & 1155 & 51 & 373 & 3.69 & 2.86 \\
\hline tRF-GIn-UUG-10-1 \#3 & GGTTTCGTAGTGTAGTGGTTAG & 22 & 689 & 84 & 565 & 102 & -3.04 & -2.46 \\
\hline tRF-GIn-UUG-1-1 \#1 & TCTGGCGACCTGGGTTCG & 18 & 78 & 1389 & 53 & 279 & 4.15 & 2.40 \\
\hline tRF-GIn-UUG-1-1 \#2 & ATCTGGCGACCTGGGTTCG & 19 & 43 & 2029 & 35 & 1014 & 5.56 & 4.84 \\
\hline tRF-Glu-CUC-1-1 \#1 & CCGTCGTAGTCTAGGTGG & 18 & 410 & 3198 & 292 & 1842 & 2.96 & 2.66 \\
\hline tRF-Glu-UUC-1-1 & TCCATTGTCGTCCAGCGG & 18 & 827 & 2866 & 1000 & 2629 & 1.79 & 1.40 \\
\hline tRF-His-AUG-1-1 \#4 & GACAGTTTGGCCGAGTGGTCT & 21 & 758 & 217 & 725 & 260 & -1.80 & -1.48 \\
\hline tRF-His-GUG-1-1 \#1 & GCCGTGGAGACCTGGGCT & 18 & 1592 & 17168 & 1167 & 12197 & 3.43 & 3.39 \\
\hline tRF-Ile-AAU-10-1 \#1 & GGCCTATTAGCTCAGCTG & 18 & 515 & 1929 & 432 & 1125 & 1.91 & 1.38 \\
\hline tRF-Ile-UAU-1-1 \#1 & CTCCCGTAGCTCAGTTGG & 18 & 26 & 778 & 8 & 306 & 4.92 & 5.20 \\
\hline tRF-iMet-CAU-10-1 \#2 & TAACCCACAGGTCCCAGGATCG & 22 & 43 & 1698 & 31 & 1006 & 5.30 & 5.04 \\
\hline tRF-Leu-AAG-10-1 \#3 & GATCAGATGGCCGAGTTGGT & 20 & 2410 & 847 & 1979 & 757 & -1.51 & -1.39 \\
\hline tRF-Lys-CUU-10-1 \#1 & CCTTGTGGTCGTGGGTTC & 18 & 79 & 971 & 42 & 675 & 3.61 & 4.00 \\
\hline tRF-Lys-CUU-10-1 \#2 & CCTTGTGGTCGTGGGTTCG & 19 & 69 & 1514 & 32 & 948 & 4.45 & 4.87 \\
\hline tRF-Lys-CUU-10-1 \#3 & AACCTTGTGGTCGTGGGTTC & 20 & 84 & 994 & 44 & 688 & 3.57 & 3.98 \\
\hline tRF-Lys-CUU-10-1 \#4 & AACCTTGTGGTCGTGGGTTCG & 21 & 301 & 6962 & 213 & 4317 & 4.53 & 4.34 \\
\hline tRF-Lys-CUU-10-1 \#5 & TAACCTTGTGGTCGTGGGTTCG & 22 & 57 & 1612 & 51 & 930 & 4.81 & 4.20 \\
\hline tRF-Lys-CUU-1-1 \#2 & GCCCGTCTAGCTCAGTCGG & 19 & 900 & 5128 & 849 & 4114 & 2.51 & 2.28 \\
\hline tRF-Lys-CUU-1-1 \#3 & GCCCGTCTAGCTCAGTCGGT & 20 & 8242 & 21424 & 3721 & 18462 & 1.38 & 2.31 \\
\hline tRF-Lys-CUU-12-1 \#1 & CCCGTCTAGCTCAGTTGG & 18 & 41 & 1279 & 21 & 484 & 4.95 & 4.53 \\
\hline tRF-Lys-CUU-12-1 \#4 & GCCCGTCTAGCTCAGTTGG & 19 & 2763 & 10617 & 2112 & 5761 & 1.94 & 1.45 \\
\hline tRF-Lys-CUU-12-1 \#2 & CCCGTCTAGCTCAGTTGGT & 19 & 230 & 5333 & 167 & 2542 & 4.54 & 3.93 \\
\hline tRF-Lys-UUU-1-1 \#1 & GCCGTCTTAGCTCAGTTGG & 19 & 154 & 807 & 123 & 432 & 2.39 & 1.81 \\
\hline tRF-Lys-UUU-2-1 \#1 & CCGACCTAGCTCAGTGGT & 18 & 174 & 883 & 103 & 806 & 2.34 & 2.97 \\
\hline tRF-Lys-UUU-2-1 \#2 & GCCGACCTAGCTCAGTGG & 18 & 1611 & 5643 & 1438 & 3708 & 1.81 & 1.37 \\
\hline tRF-Lys-UUU-3-1 \#1 & GCCGTCCTAGCTCAGTTGG & 19 & 302 & 1335 & 265 & 823 & 2.15 & 1.64 \\
\hline tRF-Met-CAU-10-1 & TCCTGAGGTCGAGAGTTC & 18 & 331 & 1750 & 256 & 1139 & 2.40 & 2.16 \\
\hline tRF-Phe-GAA-10-1 \#2 & TCTGAAGGTCGCGTGTTCG & 19 & 200 & 1396 & 123 & 873 & 2.80 & 2.83 \\
\hline tRF-Phe-GAA-10-1 \#3 & ATCTGAAGGTCGCGTGTTCG & 20 & 95 & 676 & 82 & 447 & 2.84 & 2.44 \\
\hline tRF-Phe-GAA-1-1 & GCGGGGATAGCTCAGTTG & 18 & 424 & 1119 & 226 & 1145 & 1.40 & 2.34 \\
\hline tRF-Pro-AGG-10-1 \#2 & GCGAGAGGTCCCGAGTTC & 18 & 354 & 1317 & 413 & 848 & 1.89 & 1.04 \\
\hline tRF-Pro-AGG-10-1 \#3 & GTGCGAGAGGTCCCGAGT & 18 & 74 & 640 & 75 & 750 & 3.12 & 3.32 \\
\hline tRF-Pro-CGG-1-1 & GCGAGAGGTCCCGAGTTCG & 19 & 175 & 1348 & 125 & 799 & 2.95 & 2.68 \\
\hline tRF-Thr-CGU-1-1 \#1 & CCTCCGTAGCATAGTGGT & 18 & 1256 & 4100 & 507 & 3699 & 1.71 & 2.87 \\
\hline tRF-Trp-CCA-1-1 & TCAGAAGGTTGCGTGTTCG & 19 & 192 & 1468 & 110 & 920 & 2.94 & 3.07 \\
\hline tRF-Tyr-AUA-1-1 \#2 & CCGACCTTAGCTCAGTTGG & 19 & 5560 & 18222 & 5276 & 11434 & 1.71 & 1.12 \\
\hline tRF-Val-AAC-1-1 \#2 & ACTGAAGGTCTCCGGTTCG & 19 & 59 & 524 & 46 & 420 & 3.16 & 3.20 \\
\hline tRF-Val-AAC-1-1 \#3 & CTGAAGGTCTCCGGTTCG & 18 & 66 & 892 & 61 & 620 & 3.76 & 3.34 \\
\hline tRF-Val-CAC-10-1 \#3 & GTCTGGGTGGTGTAGTTGGTTAT & 23 & 630 & 32 & 542 & 53 & -4.28 & -3.36 \\
\hline tRF-Val-CAC-10-1 \#4 & GTCTGGGTGGTGTAGTTGGTTATC & 24 & 755 & 35 & 593 & 94 & -4.42 & -2.65 \\
\hline
\end{tabular}

"tRF ID" originates from the tRNA name of wheat tRNA database (http://gtrnadb.ucsc.edu/GtRNAdb2/). The values in CSM, CSI, SMM and SMI columns were the TPM (Transcript per million reads) values over three replicates. 




selected for qRT-PCR in the four experimental groups. The expressional patterns of the majority of the target genes were highly consistent with transcriptome data (Fig. 7a). To further determine whether the target genes were regulated by tRFs in vivo, 5' RNA Ligase-Mediated Rapid Amplification of cDNA Ends (5' RLM-RACE) was conducted to capture the degraded products of the target mRNAs, which validated one of the $t R F^{\mathrm{iMet}} \operatorname{tar}$ gets and two of the tRF ${ }^{\text {Lys }}$ targets (Figure. 7b).

All of above results indicated that tRFs probably interfered with the normal cell metabolism through inhibiting their targets.

\section{Discussion}

Transfer RNA (tRNA) was traditionally considered to be a hub adaptor that transfers amino acids and helps ribosomes to decode messenger RNA $[57,58]$. Therefore, the damage of tRNA will be disastrous for a cell. Previous studies showed that tRNA can be cleaved by DICER1 in animal or RNase T2 in planta [26,59-61]. The cleaved fragment can inhibit mRNA transcriptional level, resembling the miRNA-like mechanism [18]. According to our data in wheat, the total counts of tRFs were higher than those of miRNAs especially after being infected by F. graminearum (Fig. 1a). The majority of tRFs ranged from 18 to $21 \mathrm{nt}$ in sizes, which were compatible with the lengths of miRNAs (Fig. S3). Therefore, tRFs are expected to play important roles in planta. The size distribution and the corresponding abundance also indicated that not every tRF plays function like miRNA, and the generation of tRFs was under control instead of random degradation (Fig. 1b) during F. graminearum infection process of wheat. Recent study showed that RNase T2 is involved in the response to pathogens [37], and an earlier study showed that RNase T2 was a major driver of tRFs biogenesis in plant [26]. Moreover, all of the TaDCL1 family members were dramatically inhibited by $F$ graminearum infection (Fig. S6), indicating TaDCL1 might not 

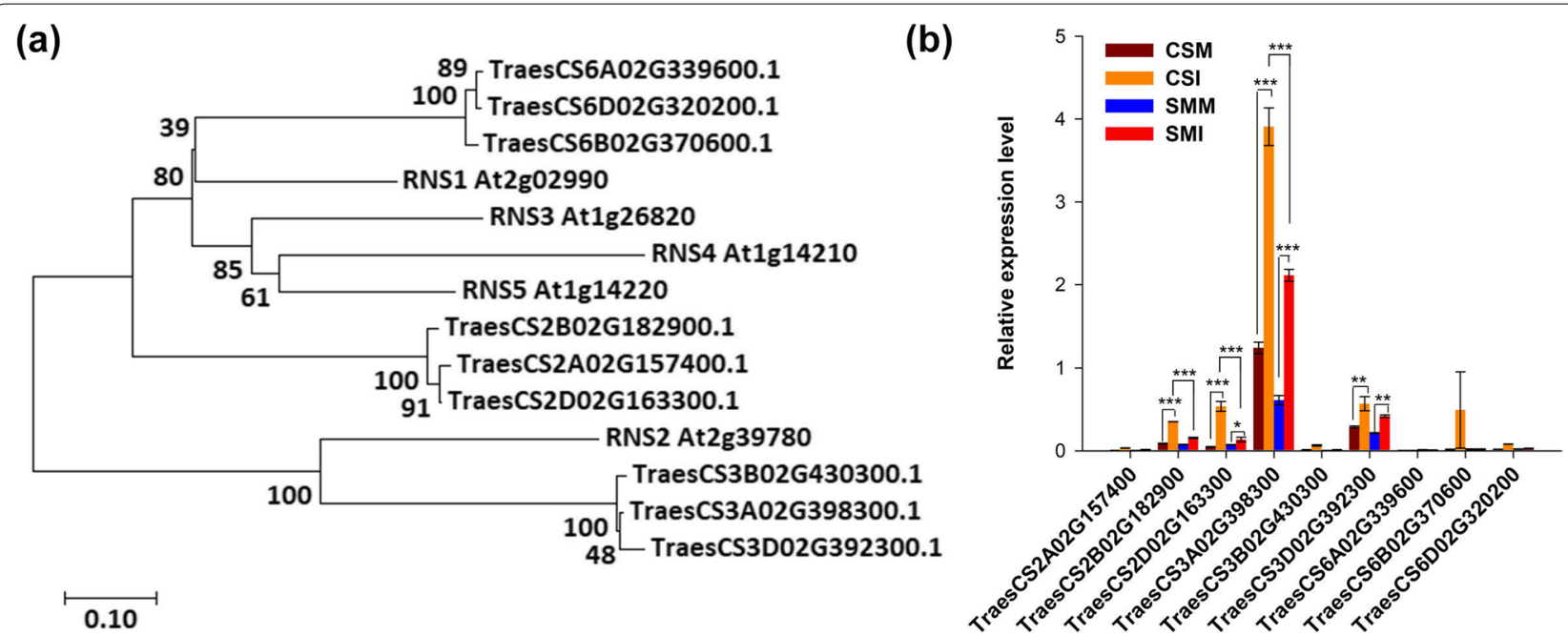

Fig. 4 RNaseT2 family members in wheat. (a) Phylogenetic tree of RNAase T2 proteins in wheat and Arabidopsis. The Neighbor-Joining (NJ) tree was generated using MEGA7 with 1000 bootstrap replicates. (b) Expressional detection of TaRNase T2 family members by qRT-PCR. Expressional values are shown as means \pm standard errors (s.e.) over three replicates. Expressional levels are calculated in $2^{-\Delta \Delta C t}$. The TaActin was used as a loading control. Student's $t$-test was used for significant difference, ${ }^{* * *}, P<0.001 ;{ }^{* *}, P<0.01 ;{ }^{*}, P<0.05$

participate in the biosynthesis of tRF. The consistent expressional patterns between RNase T2s and tRFs suggests RNase T2 most likely mediated the biogenesis of tRFs when wheat is challenged by $F$. graminearum infection (Fig. S3, Fig. 4). According to the existing research, DON prevents the elongation of peptide chain on ribosome [4], which could be the main factor causing tRNA accumulation after $F$. graminearum infection. Due to the high resistance of SM to $\mathrm{FHB}$, the number of $F$. graminearum infected spikelets in SM was much less than that of CS in the same time after inoculation, and DON content in SM was also much lower than that in CS, therefore, the abundance of $\mathrm{tRF}$ in SM was less than that in CS. In addition, the expression of RNase T2 was significantly higher in CS than that in SM (Fig. 4b). The positive correlation in expression abundance between RNase T2 and tRF suggested that the accumulation of tRFs was not only regulated by DON, but also was directly regulated by RNase T2.

Although a lot of tRFs induced by $F$. graminearum were wheat genotype-specific, especially in FHB-susceptible variety, the abundance of these specific tRFs were extremely low, most of which were less than 100 TPM (Table S3). Hence, the highly abundant and differentially expressed tRFs should be prioritized. But high abundance of tRFs doesn't mean that they respond to $F$. graminearum infection, for instance, the most enriched $t R F^{G l u(C U C-1-1 \# 3)}$ did not have significant differences between $F$. graminearum and mock inoculations (Fig. 1d, Table S2, S4). Totally eight tRFs derived from RNA-Lys-CUU-12-1 (Table S4), and three of them,

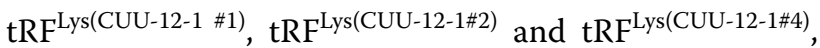
were significantly differentially expressed between $F$. graminearum and mock inoculations (Table 1). Although tRF $^{\text {Lys(CUU-12-1\#5) }}$ had the highest abundance, no significant differences were observed either between CS and SM or between F. graminearum and mock inoculations (Table S4). The mature sequences of the aforementioned four tRF ${ }^{\text {Lys }}$ were highly similar (Table S4.). Another interesting finding was that not every tRF identified here responded to $F$. graminearum infection, such as $\mathrm{tRF}^{\mathrm{Asp}}$, $\mathrm{tRF}^{\mathrm{Arg}}, \mathrm{tRF}^{\mathrm{Gly}}$ and $\mathrm{tRF}^{\text {Ser }}$ (Table 1, Table S4).

The function of tRFs is one of the most important topics of interest. According to the published work, tRFs can bind to AGO protein to form RNA-induced silencing complex (RISC) to inhibit the targets by resembling miRNA function [62]. The majority of the predicted targets for induced tRFs were downregulated after $F$. graminearum invasion (Fig. $5 \mathrm{a}$ ). These targets play pivotal roles in stress response, energy metabolism, cell component and protein digestion (Table 2, Fig. 5b-

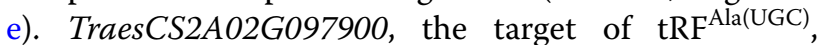
was the key polymerase in DNA replication. TraesCS2D02G337300 and TraesCS2A02G325400 potentially targeted by $t R F^{\mathrm{iMet}(\mathrm{CAU})}$ regulate the protein biosynthesis as translation initiation factor 4B. tRNA-iMet-CAU transfers the initiation amino acid methionine, and it is the most important tRNA for protein biosynthesis. Here, we found the tRFs from tRNA-iMet-CAU might play a role in regulating initiation of translation based on the annotation of $\mathrm{tRF}^{\mathrm{iMet}}$ targets, TraesCS2D02G337300 and TraesCS2A02G325400, which encode translation 
(a)

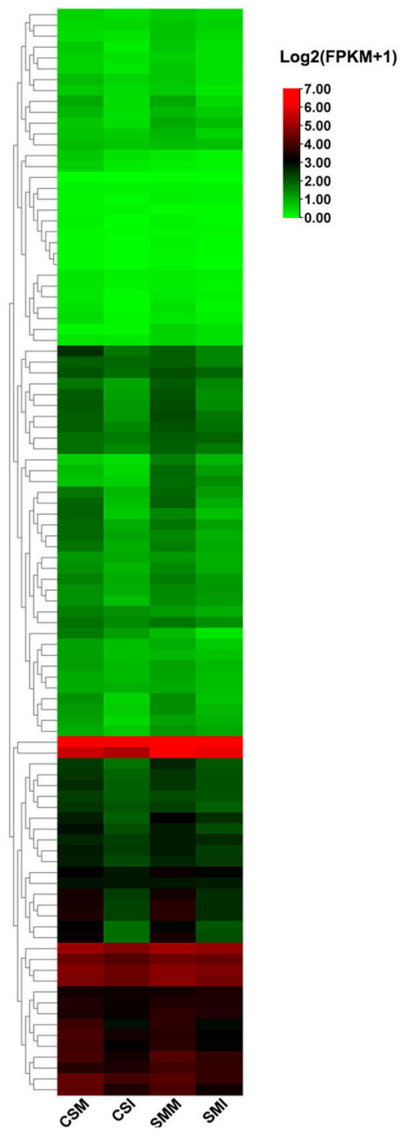

(d)

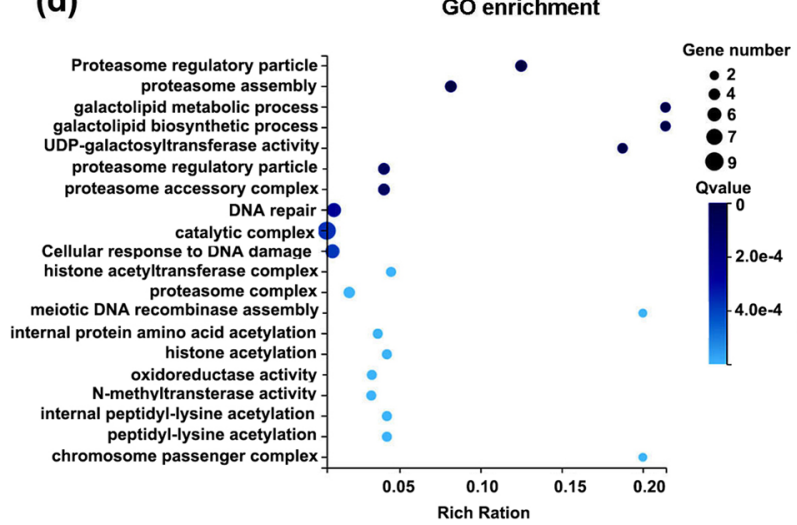

(c) (b) GO classification
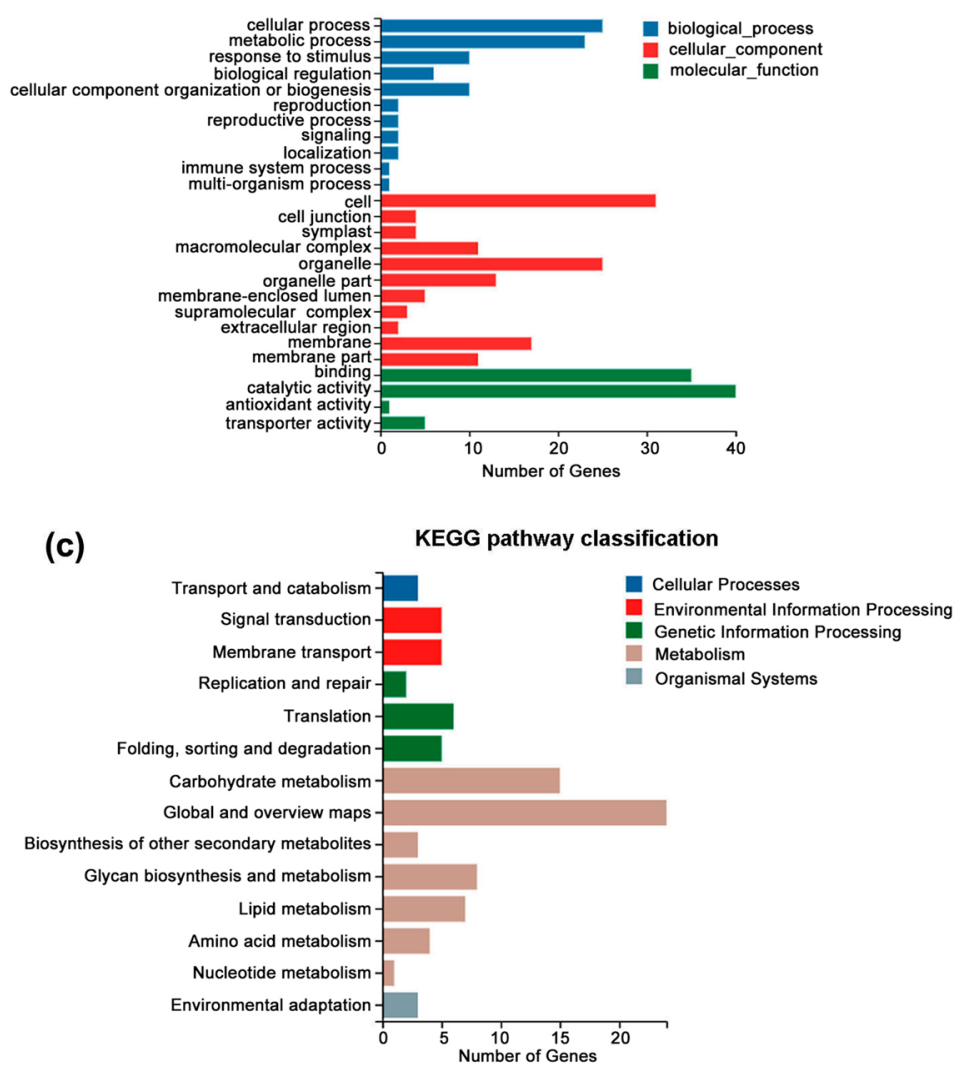

(e)

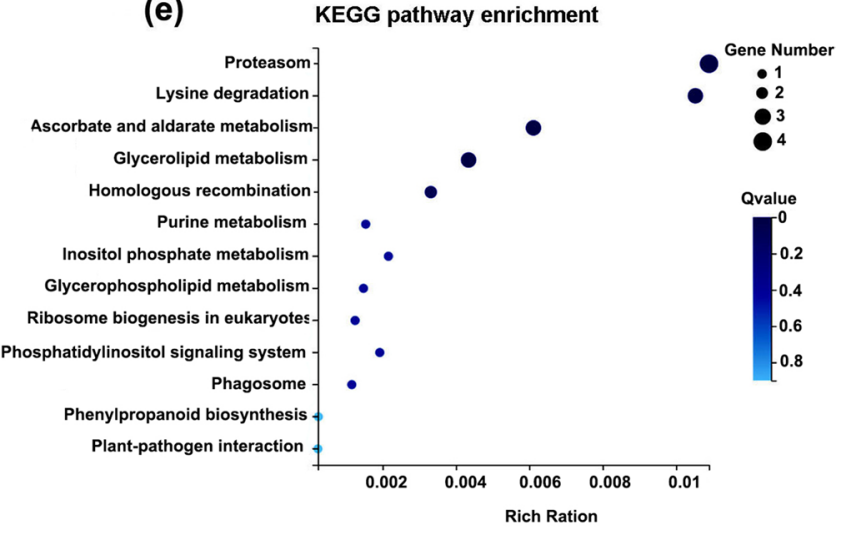

Fig. 5 Analyses of expression, GO and KEGG of differentially expressed tRFs'target genes predicted in wheat. (a) Heatmap of expressions of differentially expressed tRFs' target genes. The value is normalized from the average expression level for each row using ZeroToOne scale method. (b) GO classification analysis of the 100 predicted tRFs' target genes. (c) KEGG pathway classification of the 100 predicted tRFs' targets. (d) Scatter plot of GO enrichment of the 100 predicted tRF targets. (e) Scatter plot of KEGG pathway enrichment of the 100 predicted tRFs'targets. The enrichment ratio is measured by the number of the genes to the number of background genes in a particular GO term or KEGG pathway. The size of the dots represents the number of genes, and the color of the dots represents the range of the Q-value

initiation factor $4 \mathrm{~B}$. $\mathrm{tRF}^{\mathrm{Lys}(\mathrm{CUU})}$ might inhibit the function of E3 ubiquitin-protein ligase by cleaving its target genes TraesCS1A02G341400, TraesCS1D02G343700 and TraesCS1B02G354100, which play pivotal roles at the first step of protein degradation. ATP-binding cassette $(\mathrm{ABC})$ transporter proteins are the important channel system for material communication in cytomembrane by carrying diverse substrates across cell membranes 
Table 2 The predicted targets of highly induced tRFs by F. graminearum

\begin{tabular}{|c|c|c|c|c|c|c|c|}
\hline tRF & Target_Acc. & Exp. & $\log 2(\mathrm{CSI} / \mathrm{CSM})$ & $p$ value & $\log 2(\mathrm{SMI} / \mathrm{SMM})$ & $p$ value & Annotation \\
\hline tRF $^{\text {Ala(CGC) }}$ & TraesCS6D02G244300 & 2.5 & -0.45 & 0.5722 & -0.25 & 0.2465 & Dual specificity protein kinase YAK1 \\
\hline tRF $^{\text {Ala(CGC) }}$ & TraesCS4B02G385200 & 2.5 & -1.09 & 0.0553 & -1.05 & 0.0370 & Homeobox-leucine zipper protein \\
\hline $\operatorname{tRF}^{\mathrm{Ala}(U G \mathrm{C})}$ & TraesCS2A02G097900 & 2.5 & -0.71 & 0.0304 & -0.24 & 0.2946 & DNA-directed primase/polymerase protein \\
\hline tRF $^{\text {Ala(UGC) }}$ & TraesCS2B02G113600 & 2.5 & -0.80 & 0.0752 & -0.92 & 0.0008 & DNA-directed primase/polymerase protein \\
\hline $\operatorname{tRF}^{\mathrm{Cys}(\mathrm{GCA})}$ & TraesCS7B02G203400 & 2 & -2.64 & 0.0024 & -1.93 & 0.0025 & ABC transporter B family member 19-like \\
\hline $\operatorname{tRF}^{\mathrm{Cys}(G C A)}$ & TraesCS7D02G298600 & 2 & -2.26 & 0.0432 & -1.25 & 0.0038 & ABC transporter B family member 19-like \\
\hline $\operatorname{tRF}^{\mathrm{Cys}(\mathrm{GCA})}$ & TraesCS6B02G170700 & 2 & -0.68 & 0.0001 & -0.57 & 0.0133 & ABC transporter B family member 19-like \\
\hline $\operatorname{tRF}{ }^{C y s}(\mathrm{GCA})$ & TraesCS6D02G131900 & 2 & -1.76 & 0.0045 & -1.07 & 0.0029 & ABC transporter B family member 19-like \\
\hline $\mathrm{tRF}^{\mathrm{Glu}(\mathrm{CUC})}$ & TraesCS6B02G032300 & 2.5 & -1.34 & 0.0015 & -0.68 & 0.1562 & Pentatricopeptide repeat-containing protein \\
\hline 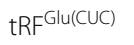 & TraesCS6D02G026900 & 2.5 & -0.82 & 0.0046 & -0.69 & 0.0073 & Pentatricopeptide repeat-containing protein \\
\hline $\mathrm{tRF}^{\mathrm{Glu}(\mathrm{CUC})}$ & TraesCS3A02G085700 & 2.5 & -0.75 & 0.0286 & -0.35 & 0.3106 & Trehalose 6-phosphate phosphatase \\
\hline tRF Glu(CUC) & TraesCS3D02G085800 & 2.5 & -0.77 & 0.0572 & -0.95 & 0.0630 & Trehalose 6-phosphate phosphatase \\
\hline 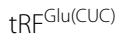 & TraesCS6D02G395700 & 2.5 & -0.93 & 0.1064 & -0.48 & 0.1659 & Interleukin-1 receptor-associated kinase 4 \\
\hline tRF Glu(CUC) & TraesCS5A02G002500 & 2.5 & -2.40 & 0.0101 & -0.64 & 0.2172 & 1-phosphatidylinositol-4-phosphate 5-kinase \\
\hline 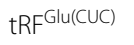 & TraesCS5D02G002700 & 2.5 & -2.47 & 0.0567 & -1.29 & 0.0146 & 1-phosphatidylinositol-4-phosphate 5-kinase \\
\hline $\operatorname{tRF} \mathrm{His}^{(\mathrm{AUG})}$ & TraesCS7B02G416600 & 2 & -1.51 & 0.0241 & -0.17 & 0.6981 & Disease resistance protein RPM1 \\
\hline $\mathrm{tRF}^{\mathrm{His}(\mathrm{AUG})}$ & TraesCS6A02G222000 & 2.5 & -0.40 & 0.1980 & -0.64 & 0.0465 & Phytosulfokine receptor 1 \\
\hline $\operatorname{tRF}^{H i s(A \cup G)}$ & TraesCS6B02G291900 & 2.5 & -1.37 & 0.0137 & -0.97 & 0.0527 & ABC transporter B family member 2-like \\
\hline $\operatorname{tRF}^{\mathrm{iMet}(\mathrm{CAU})}$ & TraesCS2B02G598800 & 2.5 & -0.88 & 0.0015 & -0.79 & 0.0010 & Polyadenylate-binding protein 4-like \\
\hline tRF $F^{\mathrm{iMet}(\mathrm{CAU})}$ & TraesCS2B02G045400 & 2.5 & -0.50 & 0.0429 & -0.49 & 0.0229 & Disease resistance RPP13-like protein 1 \\
\hline tRF $F^{\mathrm{iMet}(\mathrm{CAU})}$ & TraesCS2A02G030000 & 2.5 & -1.18 & 0.0081 & -0.91 & 0.0028 & Disease resistance RPP13-like protein 1 \\
\hline tRF ${ }^{\mathrm{iMet}(\mathrm{CAU})}$ & TraesCS2B02G044900 & 2.5 & -0.79 & 0.0047 & -0.49 & 0.0058 & Disease resistance RPP13-like protein 1 \\
\hline tRF ${ }^{\mathrm{iMet}(\mathrm{CAU})}$ & TraesCS2A02G029800 & 2.5 & -0.43 & 0.0097 & -0.34 & 0.0460 & Disease resistance RPP13-like protein 1 \\
\hline $\mathrm{tRF}^{\mathrm{iMet}(\mathrm{CAU})}$ & TraesCS2D02G337300 & 2.5 & -0.28 & 0.0164 & -0.09 & 0.5289 & Translation initiation factor $4 \mathrm{~B}$ \\
\hline $\mathrm{tRF}^{\mathrm{iMet}(\mathrm{CAU})}$ & TraesCS2A02G325400 & 2.5 & -0.27 & 0.0781 & -0.15 & 0.2779 & Translation initiation factor $4 \mathrm{~B}$ \\
\hline tRF $F^{\operatorname{Leu}(A A G)}$ & TraesCS3B02G590000 & 2 & -0.24 & 0.0213 & -0.26 & 0.0180 & UDP-D-apiose/UDP-D-xylose synthase \\
\hline tRF $F^{\text {Lys (CUU) }}$ & TraesCS6A02G164200 & 2 & -0.37 & 0.0065 & -0.24 & 0.0506 & Phosphatidylcholine transfer protein SFH2-like \\
\hline tRF $F^{\text {Lys }(\mathrm{CUU})}$ & TraesCS1D02G264300 & 2.5 & -0.71 & 0.0127 & -0.45 & 0.0158 & Ubinuclein \\
\hline tRF ${ }^{\text {Lys }(\mathrm{CUU})}$ & TraesCS6D02G254900 & 2.5 & -1.31 & 0.0004 & -1.06 & 0.0001 & Histone-lysine N-methyltransferase SUVR5 \\
\hline tRF $F^{\text {Lys (CUU) }}$ & TraesCS6A02G274600 & 2.5 & -1.11 & 0.0007 & -1.05 & 0.0001 & Histone-lysine N-methyltransferase SUVR5 \\
\hline tRF Lys(CUU) & TraesCS6B02G302100 & 2.5 & -1.94 & 0.0010 & -1.45 & 0.0006 & Histone-lysine N-methyltransferase SUVR5 \\
\hline tRF $F^{\text {Lys }(C U U)}$ & TraesCS6D02G041400 & 2.5 & -1.43 & 0.1912 & -1.23 & 0.1284 & Disease resistance protein RPS2 \\
\hline tRF ${ }_{\text {Lys }(\mathrm{CUU})}$ & TraesCS6B02G341700 & 2.5 & -1.00 & 0.0183 & -0.70 & 0.0056 & TORTIFOLIA1-like protein 3 \\
\hline tRF $F^{\text {Lys }(\mathrm{CUU})}$ & TraesCS4D02G211200 & 2.5 & -0.43 & 0.0111 & -0.45 & 0.0164 & Digalactosyldiacylglycerol synthase \\
\hline tRF $F^{\text {Lys (CUU) }}$ & TraesCS4A02G093900 & 2.5 & -0.71 & 0.0031 & -0.53 & 0.0124 & Digalactosyldiacylglycerol synthase \\
\hline tRF $F^{\text {Lys }(C U U)}$ & TraesCS4B02G210400 & 2.5 & -1.74 & 0.0005 & -1.21 & 0.0002 & Digalactosyldiacylglycerol synthase \\
\hline tRF $F^{\text {Lys }(C U U)}$ & TraesCS1A02G341400 & 1 & -1.04 & 0.2031 & -0.31 & 0.5059 & E3 ubiquitin-protein ligase \\
\hline tRF $F^{\text {Lys }(\mathrm{CUU})}$ & TraesCS1D02G343700 & 2.5 & -1.55 & 0.1171 & -0.38 & 0.4265 & E3 ubiquitin-protein ligase \\
\hline tRF Lys(CUU) & TraesCS1B02G354100 & 0 & -1.33 & 0.0733 & -0.94 & 0.0969 & E3 ubiquitin-protein ligase \\
\hline $\operatorname{tRF}^{\operatorname{Lys}(\mathrm{UUU})}$ & TraesCS4B02G253500 & 2.5 & -1.39 & 0.0016 & -0.93 & 0.0001 & Alpha-L-fucosidase 2 \\
\hline tRF $F^{\text {Lys (UUU) }}$ & TraesCS4B02G253300 & 2.5 & -0.80 & 0.0027 & -0.66 & 0.0004 & Alpha-L-fucosidase 2 \\
\hline tRF $F^{\operatorname{Lys}(U \cup U)}$ & TraesCS4D02G253400 & 2.5 & -1.05 & 0.0008 & -0.74 & 0.0028 & Alpha-L-fucosidase 2 \\
\hline tRF $F^{\operatorname{Lys}(U \cup U)}$ & TraesCS4A02G051000 & 2.5 & -0.80 & 0.0068 & -0.76 & 0.0018 & Alpha-L-fucosidase 2 \\
\hline 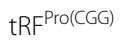 & TraesCS5A02G096600 & 0.5 & -0.19 & 0.3818 & -0.12 & 0.5391 & Mitochondrial carrier protein \\
\hline $\mathrm{tRF}^{\text {Pro(CGG) }}$ & TraesCS7B02G437300 & 2.5 & -0.53 & 0.0923 & -0.54 & 0.0429 & Disease resistance protein RPM13 \\
\hline $\operatorname{tRF}^{\operatorname{Thr}(\mathrm{CGU})}$ & TraesCS2B02G167000 & 1.5 & -1.31 & 0.0001 & -1.18 & 0.0008 & Transcription-associated protein \\
\hline $\operatorname{tRF}^{\operatorname{Thr}(\mathrm{CGU})}$ & TraesCS2A02G141800 & 1.5 & -1.40 & 0.0001 & -1.18 & 0.0005 & Transcription-associated protein \\
\hline $\operatorname{tRF}^{\operatorname{Thr}(\mathrm{CGU})}$ & TraesCS2D02G145300 & 1.5 & -1.77 & 0.0001 & -1.47 & 0.0012 & Transcription-associated protein \\
\hline $\mathrm{tRF}^{\operatorname{Th} r(\mathrm{CGU})}$ & TraesCS3D02G364200 & 2.5 & -0.94 & 0.0004 & -0.74 & 0.0011 & WD40 domain contain protein \\
\hline
\end{tabular}


Table 2 (continued)

\begin{tabular}{|c|c|c|c|c|c|c|c|}
\hline tRF & Target_Acc. & Exp. & $\log 2(\mathrm{CSI} / \mathrm{CSM})$ & $p$ value & $\log 2(\mathrm{SMI} / \mathrm{SMM})$ & $p$ value & Annotation \\
\hline $\operatorname{tRF}^{\operatorname{Thr}(\mathrm{CGU})}$ & TraesCS3A02G371100 & 2.5 & -0.78 & 0.1390 & -0.62 & 0.5027 & WD40 domain contain protein \\
\hline $\operatorname{tRF}^{\operatorname{Thr}(\mathrm{CGU})}$ & TraesCS1B02G075900 & 2.5 & -0.87 & 0.2030 & -0.33 & 0.6064 & DNA repair protein RAD51 like protein \\
\hline $\operatorname{tRF}^{\operatorname{Thr}(\mathrm{CGU})}$ & TraesCS1D02G059600 & 2.5 & -0.97 & 0.4057 & -0.33 & 0.6472 & DNA repair protein RAD51 like protein \\
\hline $\operatorname{tRF}^{\operatorname{Trp}(\mathrm{CCA})}$ & TraesCS5D02G256900 & 2.5 & -0.66 & 0.0037 & -0.33 & 0.0131 & tRNA (cytosine(34)-C(5))-methyltransferase \\
\hline $\mathrm{tRF}^{\operatorname{Trp}(\mathrm{CCA})}$ & TraesCS5B02G247600 & 2.5 & -0.82 & 0.0083 & -0.43 & 0.0256 & tRNA (cytosine(34)-C(5))-methyltransferase \\
\hline $\operatorname{tRF}^{\operatorname{Tyr}(\mathrm{AUA})}$ & TraesCS4A02G406400 & 0 & -0.49 & 0.0106 & -0.50 & 0.0318 & Dynein assembly factor 1 \\
\hline $\mathrm{tRF}^{\operatorname{Tyr}(\mathrm{AUA})}$ & TraesCS1B02G371300 & 2.5 & -0.43 & 0.3945 & -0.39 & 0.3175 & Alpha-1,3-arabinosyltransferase XAT2-like \\
\hline $\mathrm{tRF}^{\mathrm{Val}(\mathrm{AAC})}$ & TraesCS1D02G283000 & 1.5 & -0.73 & 0.3259 & -1.41 & 0.2318 & Laccase \\
\hline $\operatorname{tRF}^{\mathrm{Val}(\mathrm{AAC})}$ & TraesCS7B02G408700 & 2 & -2.33 & 0.1853 & -0.56 & 0.2495 & ARR transcriptional factor \\
\hline $\mathrm{tRF}^{\mathrm{Val}(\mathrm{AAC})}$ & TraesCS7B02G194500 & 2.5 & -0.82 & 0.0113 & -0.37 & 0.1357 & Galactan beta-1,4-galactosyltransferase GALS1 \\
\hline $\operatorname{tRF}^{\mathrm{Val}(\mathrm{AAC})}$ & TraesCS4A02G444300 & 2.5 & -0.89 & 0.0013 & -0.75 & 0.0003 & Phosphoglucan, water dikinase \\
\hline
\end{tabular}

The targets of tRFs were analyzed using psRNATargets analysis server (http://plantgrn.noble.org/psRNATarget/analysis). The list of tRF sequences were submitted and the cDNA library of Triticum aestivum for target search.

[63], which are the predicted targets of $\mathrm{tRF}^{\mathrm{Cys}(\mathrm{GCA})}$ and tRF $\mathrm{His}^{\mathrm{HiUG})}$ based on our study.

Several genes were reported to be associated with disease resistance, such as TraesCS7B02G416600, TraesCS2B02G045400, TraesCS6D02G041400 and TraesCS7B02G437300, which encodes disease-resistance protein RPM1 [64], RPP13-like protein [65], RPS2 [66], RPM13 [67], and they were the predicted targets of $\mathrm{tRF}^{\mathrm{His}(\mathrm{AUG})}, \mathrm{tRF}^{\mathrm{iMet}(\mathrm{CAU})}, \mathrm{tRF}^{\mathrm{Lys}(\mathrm{CUU})}$, and

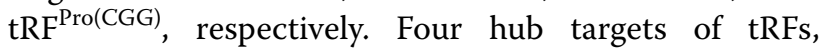
TraesCS2B02G598800, TraesCS6D02G254900, TraesCSU02G123400 and TraesCS2A02G141800, may play crucial roles in host resistance to pathogen attack (Fig. 6). TraesCS2B02G598800 encodes polyadenylatebinding protein, which was associated with ribonucleic acid (RNA) stress granule (SG) pathways [68]. TraesCS6D02G254900 encodes a histone-lysine N-methyltransferase SUVR5, which mediates H3K9me2 deposition and regulates gene expression in a DNA methylationindependent manner by regulating their chromatin and transcriptional state to rapidly adapt to environment or developmental cues [69]. TraesCSU02G123400 encodes an adenylate kinase, which plays an important role in cellular energy homeostasis and in adenine nucleotide metabolism [70]. TraesCS2A02G141800 plays a pivotal role at the level of protein transcription. In summary, these four hub genes regulate gene functions at DNA level (TraesCS6D02G254900), RNA level (TraesCS2B02G598800), protein level (TraesCS2A02G141800) and metabolic activity in cell (TraesCSU02G123400), and they were predicted to be targeted by corresponding tRFs when wheat was infected by $F$. graminearum.

The higher tRFs abundance in the susceptible variety CS may be related to its weak resistance to toxins, or the susceptible variety might induce $F$. graminearum to produce more DON toxin. The negative correlation of expression patterns between tRFs and disease resistancerelated target genes indicates that these tRFs may contribute host susceptibility to F. graminearum by silencing their target genes. The resistance level of plant to the fungus is expected to be enhanced if the tRFs could be eliminated quickly or the targets could escape the suppression by tRFs-induced silencing.

\section{Conclusion}

This is the first report of tRFs involved in wheat- $F$. graminearum interaction. One of the most important findings was that more tRFs were accumulated in FHBsusceptible variety CS than that in FHB-resistant variety SM. During infection of the wheat cells, F. graminearum secreted DON to attack wheat ribosome and inhibit the formation of peptide chain, consequently leading to accumulation of tRNA. RNase T2 was also induced by pathogen to degrade host tRNA to fragments of various sizes, and these tRFs could be assembled with AGO protein to form RNA-induced silencing complex (RISC) to inhibit the host gene expressions. Therefore, tRFs might negatively regulate wheat resistance to FHB by interfering with the normal cell metabolism, cell cycle, and some of the disease resistance genes at post transcriptional level. However, the relationships between tRFs and their targets need to be further deciphered.

\section{Methods}

Plant materials and F. graminearum inoculation experiment Wheat varieties, Chinese Spring (CS)" and "Sumai3 (SM)", were used in this study, which were kindly provided by Dr. Guihua Bai at Kansas State University, USA. CS is an FHB-susceptible variety [42, 43], and SM is a famous FHB-resistant variety which carries Fhb1, a major 


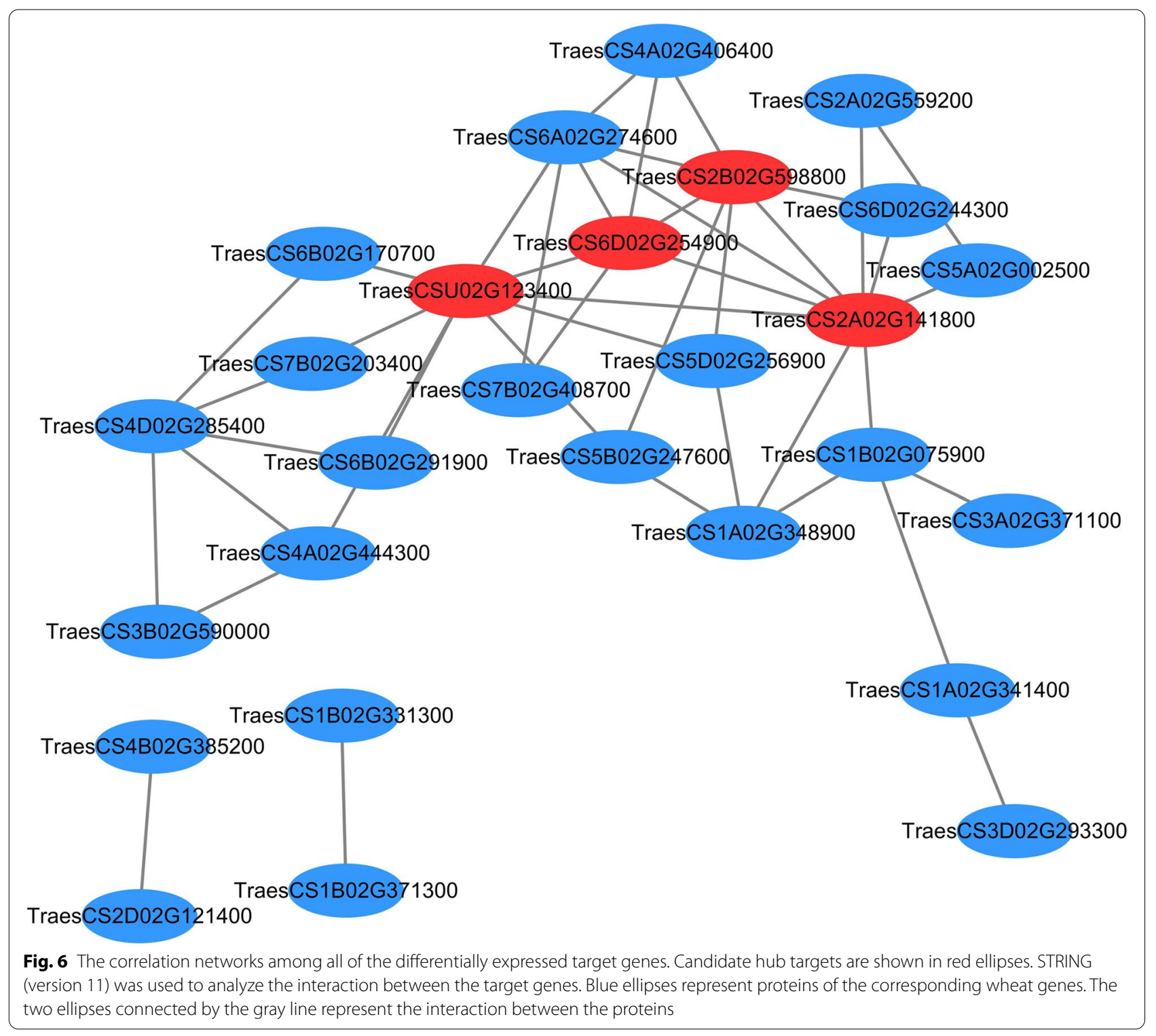

QTL conferring Type 2 resistance to FHB [44-46]. The seeds were surface sterilized by soaking in $70 \%$ ethanol for $10 \mathrm{~min}$ and then rinsed four times with sterile water. The sterilized seeds were sown in a mix of vermiculite and soil with a ratio of $1: 3$. The seedlings were grown in a condition-controlled phytotron about 3 weeks under 24 ${ }^{\circ} \mathrm{C}$ and $16 \mathrm{~h}$ of light / $8 \mathrm{~h}$ of dark cycle, and then were moved to a refrigerator at $4^{\circ} \mathrm{C}$ for vernalization for one month, and then the seedlings were moved back to the phytotron for growth.

Wheat spikes were inoculated with macroconidial spores of $F$. graminearum strain $\mathrm{PH}-1$, which was donated by Dr. Bing Li at Zhengzhou University, China. Macroconidia were produced in mung bean broth following the protocol described by Bai et al .[47]. For each wheat variety, eight spikes were inoculated at early anthesis by injecting a $10 \mu \mathrm{L}$ of the spore suspension (100 conidia $\mu \mathrm{L}^{-}$ ${ }^{1}$ ) into the two bilateral florets of the fifth spikelet from the bottom of a spike. Mock inoculation was performed as a control, where a $10 \mu \mathrm{L}$ of mung bean broth was used. The inoculated spikelets and their adjoined rachis were collected at $6 \mathrm{~h}, 12 \mathrm{~h}, 24 \mathrm{~h}, 2$ day (d), $3 \mathrm{~d}, 4 \mathrm{~d}, 5 \mathrm{~d}$, and $6 \mathrm{~d}$ post $F$. graminearum (CSI, SMI) and mock inoculations (CSM, SMM) respectively. Plants were grown in a condition-controlled phytotron under $28^{\circ} \mathrm{C}$ and $16 \mathrm{~h}$ of light / 8 h of dark cycle. Six independent biological replicates were conducted, with three biological replicates for sequencing and the remaining three for validation. 


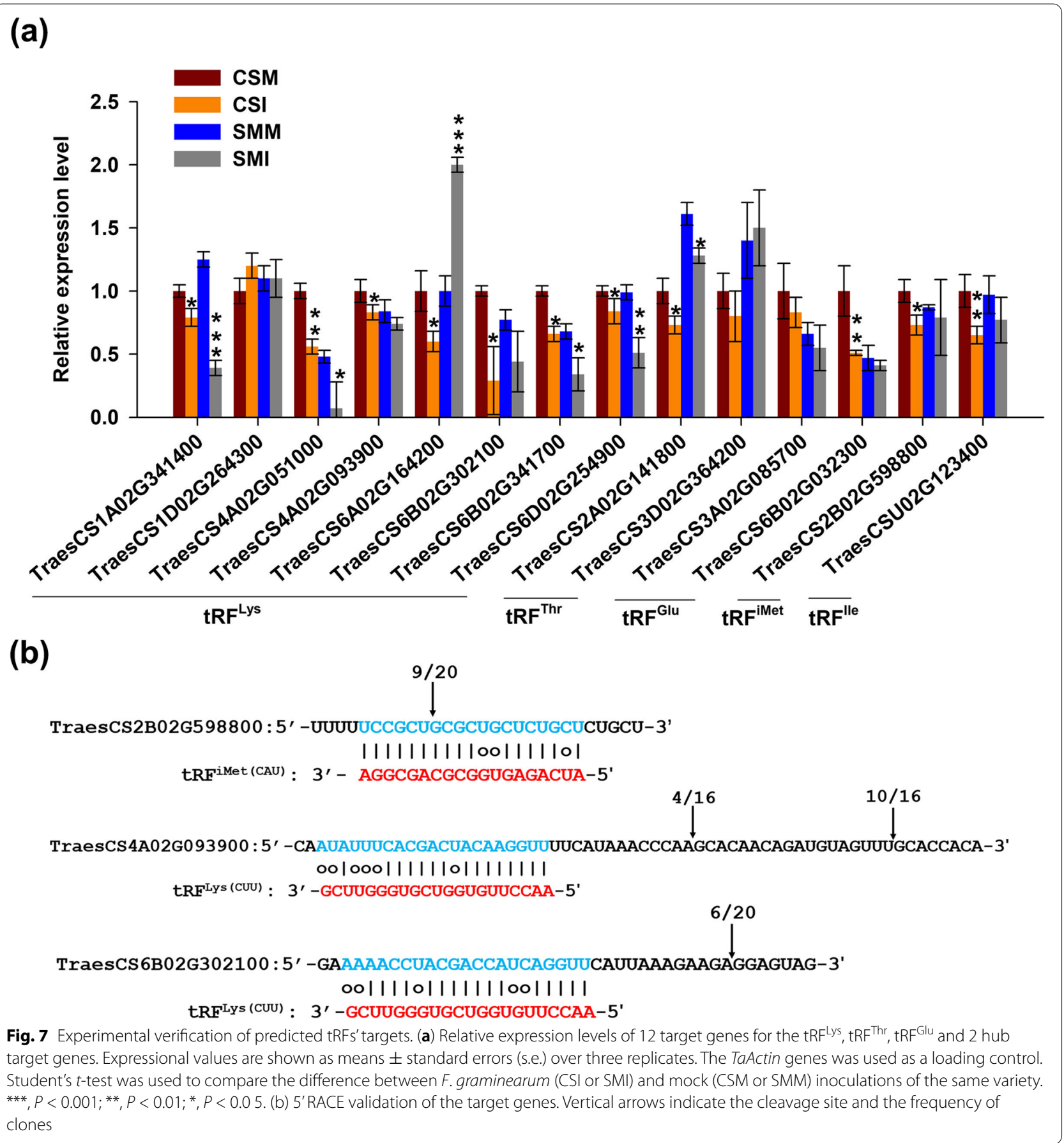

\section{RNA extraction}

A mixed sample from the eight timepoints was prepared for RNA extraction. RNAiso plus reagent (TAKARA BIO INC, Shiga, Japan) was used for purification of the total RNA from the mixed samples according to the manual instructions. The tissue samples were ground in liquid nitrogen into powder and about $80 \mathrm{mg}$ of the sample powder was then transfered into a $2 \mathrm{~mL}$ of tube with 1
$\mathrm{mL}$ of preheated RNAiso plus reagent and sufficient mixing using vortex oscillator. $200 \mu \mathrm{L}$ of chloroform was added into the mixture. After being centrifuged at 12,000 $\times \mathrm{g}$ for $10 \mathrm{~min}$ at $4{ }^{\circ} \mathrm{C}, 700 \mathrm{~mL}$ of the supernatant was transferred to a new $1.5 \mathrm{~mL}$ RNase free tube. The supernatant was mixed with an equal volume of isopropyl alcohol and placed at $-20^{\circ} \mathrm{C}$ for 1 hour for precipitation. After that, the mixture was centrifuged at $12,000 \times \mathrm{g}$ for $15 \mathrm{~min}$ 
at $4{ }^{\circ} \mathrm{C}$ and the supernatant was removed. After being washed with $1 \mathrm{~mL}$ of $75 \%$ ethanol, the RNA pellet was air-dried in the biosafety cabinet and was dissolved by 50 $\mu \mathrm{L}$ of DEPC-treated water. The quantification of the total RNA was assessed by NanoDrop 2000c spectrophotometer (Thermo Fisher Scientific, Lenexa, KS, USA), and RNA quality was checked using Agilent 2100 bioanalyzer (Thermo Fisher Scientific, MA, USA).

\section{Small RNA library construction}

A small RNA library was prepared with $1 \mu \mathrm{g}$ of the total RNA for each sample. The total RNA was purified by electrophoretic separation on a $15 \%$ urea denaturing polyacrylamide gel electrophoresis (PAGE) gel and the small RNA regions corresponding to $18-30 \mathrm{nt}$ bands in the marker lane (14-30 ssRNA Ladder Marker, TAKARA) were excised and recovered. Then the small RNAs of 18-30 nt were ligated to adenylated 3' adapters annealed to unique molecular identifiers (UMI), followed by the ligation of 5' adapters. The adapter-ligated small RNAs were subsequently transcribed into cDNA by SuperScript II Reverse Transcriptase (Invitrogen, USA) and then several rounds of PCR amplification with PCR Primer Cocktail and PCR Mix were performed to enrich the cDNA fragments. The PCR products were separated by agarose gel electrophoresis with target fragments of 110-130 bp, and then purified by QIAquick Gel Extraction Kit (QIAGEN, Valencia, CA). The distribution of the fragment sizes in the library was checked using the Agilent 2100 bioanalyzer. The library was quantifed using real-time quantitative PCR (QPCR) (TaqMan Probe). The final ligated PCR products were sequenced using the BGISEQ-500 platform (BGI-Shenzhen, China).

\section{mRNA library construction}

Oligo(dT)-attached magnetic beads were used to purify mRNA. The purified mRNA was fragmented into small pieces in a fragment buffer at appropriate temperature. Then first-strand cDNA was generated using random hexamer-primed reverse transcription, followed by a second-strand cDNA synthesis. Afterwards, A-Tailing Mix and RNA Index Adapters were added by incubating to end repair. The cDNA fragments obtained from previous step were amplified by PCR, and the products were purified by Ampure XP Beads, then were dissolved in EB solution. The product was validated on the Agilent Technologies 2100 bioanalyzer for quality control. The double stranded PCR products from the previous step were heated to be denatured and circularized by the splint oligo sequence to get the final library. The single strand circle DNA (ssCir DNA) was normalized as the final library. The final library was amplified with phi29 to make DNA nanoball (DNB), with more than 300 copies of one molecular, and the DNBs were loaded into a patterned nanoarray and single end 50 bases reads were generated on BGIseq500 platform (BGI-Shenzhen, China) [48].

\section{Data analysis}

The raw sequencing data are called raw tags. The raw tags were processed following the next steps: (1) removing low quality tags; (2) removing tags with 5 ' primer contaminants; (3) removing tags without 3' primer; (4) removing tags without insertion; (5) removing tags with poly A. The processed reads longer than $18 \mathrm{nt}$ were then mapped to the $F$. graminearum reference genome sequence (FungiDB) using Bowtie2 (v2.2.5) [49] with a parameter of "-v 0 ". The reads unmapped to the $F$. graminearum sequences were subsequently mapped to the wheat reference genome sequence (EnsemblPlants 47 release) using Bowtie2 (v2.2.5) [49]. The reads perfectly mapped to the wheat genome sequence without any mismatches were further annotated and those perfectly matching transfer RNA (tRNA) sequences of Triticum aestivum (http://gtrnadb.ucsc.edu/GtRNAdb2/) were considered as wheat tRNA derived fragments (tRFs). For miRNA and sRNA, raw reads were filtered using the fastx_toolkit (http://hannonlab.cshl.edu/ fastx_toolkit/). The clean reads of 18-29 nt in length were mapped to a structural RNA (ribosomal RNAs, transfer RNAs, small nucleolar RNAs and small nuclear RNAs) database (http://rfam.xfam.org/). Then the unmapped reads were aligned to the Triticum aestivum genome (EnsemblPlants 47 release, https://plants.ensembl.org/Triticum_aesti vum) using Bowtie. Relative abundance of unique wheat tRFs in each library was normalized to transcript per million reads (TPM).

For mRNA, the sequencing data was filtered with SOAPnuke (v1.5.2) [50] by (1) removing those reads containing sequencing adapter; (2) removing those reads with low-quality base ratio (base quality less than or equal to 5) more than 20\%; (3) removing those reads with unknown base ('N' base) ratio more than $5 \%$, afterwards clean reads were obtained and stored in FASTQ format. The clean reads were mapped to the Triticum aestivum reference genome (EnsemblPlants 47 release, https:// plants.ensembl.org/Triticum_aestivum) using HISAT2 (v2.0.4) [51]. Bowtie2 (v2.2.5) was applied to align the clean reads to the reference gene, then the expression level of a gene was calculated by RSEM (v1.2.12) [52]. Differential expression analysis was performed using the DESeq2 (v1.4.5) [53] with Q value $\leq 0.05$. GO (http:// www.geneontology.org/) and KEGG (https://www. kegg.jp/) enrichment analysis of annotated different expressed gene were performed by Phyper (https://en. wikipedia.org/wiki/Hypergeometric_distribution) based 
on Hypergeometric test. The significant levels of terms and pathways were corrected by $\mathrm{Q}$ value with a rigorous threshold $(\mathrm{Q}$ value $\leq 0.05)$ by Bonferroni [54].

\section{Prediction of target genes}

To identify the targets for the tRFs, psRNAtarget (http:// plantgrn.noble.org/psRNA Target/), a plant miRNA target finder software web tool, was used. The parameters were set to Triticum aestivum reference genome to BLAST the target sites against the tRF sequences. To annotate the target genes for tRFs which were significantly differentially expressed, Blast to Gene Ontology (Blast2GO) (http://www.blast2go.com/b2glaunch tool) was used against the $\mathrm{Nr}$ database of National Center for Biotechnology Information (NCBI) (ftp://ftp.ncbi.nih. gov/blast/db/ ).

\section{qRT-PCR validation of tRFs}

Stem-loop quantitative reverse transcription PCR (stemloop qRT-PCR) [55] was performed to evaluate relative abundance of wheat tRFs. The specificity of stem loop RT-PCR for individual tRFs was confirmed by sequencing of the amplified fragments. TaU6 gene was used as an internal reference to quantify the relative abundance of tRFs determined by stem-loop qRT-PCR from three technological replicates.

\section{5'RLM-RACE}

A mixed sample of CSI was prepared for RACE. Total RNA was extracted and purified using the method described above. A SMARTer RACE 5'/3' Kit (Takara Bio Inc., Kusatsu, Japan) was used to generate RACE products following the manufacturer's protocol. The PCR products were cloned into the pEASY-Blunt3 vector (Transgen biotech, Beijing, China) and sequenced in GENEWIZ company (Suzhou, China). Gene-specific primers was listed in Table S7.

\section{tRNA structure draw}

RNAfoldWebServer (http://rna.tbi.univie.ac.at//cgi-bin/ RNAWebSuite/RNAfold. cgi) was used to draw the tRNA primary structures of tRFs.

\section{Statistical analysis}

The charts in this study were drawn using GraphPad Prism5 and SigmaPlot 10.0 (Systat Software). SPSS19 was used for data analysis. One-way ANOVA and student's $t$ test were performed to generate $p$ values. Heatmap, volcano plot and venn chart were produced by TBtools software [56]. STRING (version 11) was used to analyze the interaction between the target genes for tRFs in wheat.

\section{Abbreviations}

FHB: Fusarium head blight; tRNAs: transfer RNAs; tRFs: tRNA-derived fragments; DCL1: Dicer-like protein 1; miRNA: microRNA; RISC: RNA-induced silencing complex; CS: Chinese Spring; SM: Sumai3; DON: deoxynivalenol; QTL: quantitative trait loci; CSM: Chinese Spring inoculated with mock; CSI: Chinese Spring inoculated with F. graminearum; SMM: Sumai3 inoculated with mock; SMI: Sumai3 inoculated with F. graminearum; TPM: transcripts per million reads; DPI: days post inoculation; RNS: S-like RNases.

\section{Supplementary Information}

The online version contains supplementary material available at https://doi. org/10.1186/s12870-021-03393-9.

Additional file 1: Figure S1. FHB disease symptoms of Chinese spring (CS) and Sumai 3 (SM). (a) Photos were taken at $10 \mathrm{~d}$ after inoculation with F. graminearum. The arrow in yellow indicates the spikelet inoculated with F. graminearum. CSM, Chinese Spring (CS) with mock inoculation; CSI, CS with F. graminearum inoculation; SMM, Sumai3 (SM) with mock inoculation; SMI, SM with F. graminearum inoculation. Bar $=2 \mathrm{~cm}$. (b) Number of diseased spikelets scored at $10 \mathrm{~d}$ after inoculation. Student's $t$-test was used to compare the difference between CSI and SMI.

Additional file 2: Figure S2. Overview of tRFs and tRNA matching diagrams.

Additional file 3: Figure S3. Abundance and size of the wheat tRFs under mock and F. graminearum inoculations. The $x$-axis indicates the size of the major wheat tRFs ranging from 18 to $28 \mathrm{nt}$ and the $y$-axis indicates the means of normalized tag counts of different sizes of the tRFs from small RNA-Seq.

Additional file 4: Figure S4. The expressional patterns of $\mathrm{tRF}^{\mathrm{Glu}}$, $\mathrm{tRF}^{\mathrm{Lys}}$ and $\mathrm{RF}^{\mathrm{Thr}}$ at different time points. (a), (b) and (c) show the expressional levels of corresponding tRF in time course by stem-loop-qRT-PCR. The $x$-axis represents the sample collecting time after inoculation with F. graminearum; TPI, time post inoculation. The $y$-axis represents the expressional level of the tRF at each time point. Expressional values are calculated in $2^{-\Delta \Delta C t}$. The snRNA gene TaU6 was used as a loading control. Similar results were obtained from three biological repeats.

Additional file 5: Figure S5. The expression profiles of TaRNase T2 family members by RNA-Seq. Expression levels are shown as means \pm standard errors (s.e.) over three biological replicates. Student's $t$-test was used for difference analysis, ${ }^{* *}, P<0.001 ;{ }^{*}, P<0.05$.

Additional file 6: Figure S6.The expression profiles of TaAGO1 and TaDCL family members by RNA-seq. Expression levels are shown as means \pm standard errors (s.e.) over three biological replicates. Student's t-test was used for difference analysis, ${ }^{* * *}, P<0.001$.

Additional file 7: Table S1. Statistics of sequencing libraries

Additional file 8: Table S2. Summary of the identified tRFs

Additional file 9: Table S3. Lists of specific tRFs in different groups

Additional file 10: Table S4. List of highly expressed tRFs

Additional file 11: Table S5. Summary of fragments derived from tRNA

Additional file 12: Table S6. The list of tRFs'targets in wheat

Additional file 13: Table S7. The primers used in this study

Additional file 14.

\section{Acknowledgements}

We thank Dr. Guihua Bai at Kansas State University, USA, for kindly providing the wheat varieties and Dr. Bing Li at Zhengzhou University, China, for donating the Fusarium graminearum isolate $\mathrm{PH}-1$. We also thank the Editors and the two anonymous reviewers for constructive comments to improve the manuscript.

\section{Authors' contributions}

$\mathrm{ZS}$ and $\mathrm{TL}$ contributed to the idea conception and experimental design. YH and $Y Z$ collected the samples for sRNA-sequencing and RNA-sequencing. ZS 
and LL performed data analysis. YH, SH and NJ performed qRT-PCR validation. $\mathrm{ZS}$ and TL wrote the manuscript. All authors read and approved the final manuscript.

\section{Funding}

This work was supported by the National Key R\&D Program: Intergovernmental Key Items for International Scientific and Technological Innovation Cooperation (2018YFE0107700), China Postdoctoral Science Foundation (2019M661949), Natural Science Fund for Colleges and Universities in Jiangsu Province (20KJB210011), National Natural Science Foundation of China (31771772), and the Priority Academic Program Development of Jiangsu Higher Education Institutions (PAPD). All these funds support the sRNAsequencing, RNA-sequencing, RACE and qRT-PCR validation.

\section{Availability of data and materials}

The data that support the findings of this study are available from the corresponding author upon reasonable request. All sequences generated by sequencing in this study can be found in the NCBI Short Reads Archive (SRA) BioProject PRJNA683746 (https://www.ncbi.nlm.nih.gov/sra/?term=PRJNA 683746)

\section{Declarations}

\section{Ethics approval and consent to participate}

Not applicable.

\section{Consent for publication}

Not applicable.

\section{Competing interests}

The authors declare that they have no competing interests

Received: 27 July 2021 Accepted: 9 December 2021

Published online: 03 January 2022

\section{References}

1. Bai G, Shaner G. Management and resistance in wheat and barley to Fusarium head blight. Annu. Rev. Phytopathol. 2004;42(1):135-61.

2. Dean R, Van Kan JAL, Pretorius ZA, Hammond-Kosack KE, Di Pietro A, Spanu PD, et al. The Top 10 fungal pathogens in molecular plant pathology. Mol. Plant Pathol. 2012;13(7):804-4.

3. Chen Y, Kistler HC, Ma Z. Fusarium graminearum Trichothecene Mycotoxins: Biosynthesis, Regulation, and Management. Annu. Rev. Phytopathol. 2019:57:15-39.

4. Garreau de Loubresse N, Prokhorova I, Holtkamp W, Rodnina MV, Yusupova G, Yusupov M. Structural basis for the inhibition of the eukaryotic ribosome. Nature. 2014;513(7519):517-22.

5. Minervini F, Fornelli F, Flynn KM. Toxicity and apoptosis induced by the mycotoxins nivalenol, deoxynivalenol and fumonisin B1 in a human erythroleukemia cell line. Toxicology in Vitro 2004, 18(1):0-28.

6. Diesing A, Nossol C, Dänicke S, Walk N, Post A, Kahlert S, et al. Vulnerability of polarised intestinal porcine epithelial cells to mycotoxin deoxynivalenol depends on the route of application. PLoS One. 2011;6(2):e17472.

7. Pestka JJ. Deoxynivalenol-Induced Proinflammatory Gene Expression: Mechanisms and Pathological Sequelae. Toxins. 2010;2(6):1300-17.

8. Rocha O, Ansari K, Doohan FM. Effects of trichothecene mycotoxins on eukaryotic cells: A review. Food Addit. Contam. 2005;22(4):369-78.

9. Rawat N, Pumphrey MO, Liu S, Zhang X, Tiwari VK, Ando K, et al. Wheat Fhb1 encodes a chimeric lectin with agglutinin domains and a poreforming toxin-like domain conferring resistance to Fusarium head blight. Nat. Genet. 2016;48(12):1576-80.

10. Su Z, Bernardo A, Tian B, Chen H, Wang S, Ma H, et al. A deletion mutation in TaHRC confers Fhb1 resistance to Fusarium head blight in wheat. Nat. Genet. 2019;51:1099-105.

11. Li G, Zhou J, Jia H, Gao Z, Fan M, Luo Y, et al. Mutation of a histidine-rich calcium-binding-protein gene in wheat confers resistance to Fusarium head blight. Nat. Genet. 2019;51:1106-12.
12. Wang H, Sun S, Ge W, Zhao L, Hou B, Wang K, et al. Horizontal gene transfer of Fhb7 from fungus underlies Fusarium head blight resistance in wheat. Science. 2020;368:6493.

13. Guo X, Shi Q, Yuan J, Wang M, Wang J, Wang C, et al. Alien chromatin but not Fhb7 confers Fusarium head blight resistance in wheat breeding. In.: bioRxiv. 2021.

14. Gu C, Begley TJ, Dedon PC. tRNA modifications regulate translation during cellular stress. FEBS Lett. 2014;588(23):4287-96.

15. Honda S, Loher P, Shigematsu M, Palazzo JP, Suzuki R, Imoto I, et al. Sex hormone-dependent tRNA halves enhance cell proliferation in breast and prostate cancers. Proc. Natl. Acad. Sci. 2015;112(29):E3816-25.

16. Lyons SM, Fay MM, Ivanov P. The role of RNA modifications in the regulation of tRNA cleavage. FEBS Lett. 2018;592(17):2828-44.

17. Cole C, Sobala A, Lu C, Thatcher SR, Bowman A, Brown JW, et al. Filtering of deep sequencing data reveals the existence of abundant Dicerdependent small RNAs derived from tRNAs. RNA. 2009;15(12):2147-60.

18. Li S, Xu Z, Sheng J. tRNA-derived small RNA: A novel regulatory small non-coding RNA. Genes. 2018;9(5):246.

19. Ma X, Liu C, Kong X, Liu J, Zhang S, Liang S, et al. Extensive profiling of the expressions of tRNAs and tRNA-derived fragments (tRFs) reveals the complexities of tRNA and tRF populations in plants. Sci. China Life Sci. 2021;64:495-511.

20. Yamasaki S, Ivanov P, Hu GF, Anderson P. Angiogenin cleaves tRNA and promotes stress-induced translational repression. J. Cell Biol. 2009;185(1):35-42.

21. Thompson DM, Parker R. The RNase Rny $1 p$ cleaves tRNAs and promotes cell death during oxidative stress in Saccharomyces cerevisiae. J. Cell Biol. 2009;185(1):43-50.

22. Andersen KL, Collins K. Several RNase T2 enzymes function in induced tRNA and rRNA turnover in the ciliate Tetrahymena. Mol. Biol. Cell. 2011:23(1):36-44

23. Li Z, Ender C, Meister G, Moore PS, Chang Y, John B. Extensive terminal and asymmetric processing of small RNAs from rRNAs, snoRNAs, snRNAs, and tRNAs. Nucleic Acids Res. 2012;40(14):6787-99.

24. Kumar P, Anaya J, Mudunuri SB, Dutta A. Meta-analysis of tRNA derived RNA fragments reveals that they are evolutionarily conserved and associate with AGO proteins to recognize specific RNA targets. BMC Biol. 2014;12:78-8

25. German M, Choudury SG, Keith SR. tRNA-derived small RNAs target transposable element transcripts. Nucleic Acids Res. 2017:9:9.

26. Megel C, Hummel G, Lalande S, Ubrig E, Cognat V, Morelle G, et al. Plant RNases T2, but not Dicer-like proteins, are major players of tRNA-derived fragments biogenesis. Nucleic Acids Res. 2019;47(2):941-52.

27. Maute RL, Schneider C, Sumazin P, Holmes A, Califano A, Basso K, et al. tRNA-derived microRNA modulates proliferation and the DNA damage response and is down-regulated in B cell lymphoma. Proc. Natl. Acad. Sci. 2013;110(4):1404-9.

28. Kawamura Y, Saito K, Kin T, Ono Y, Asai K, Sunohara T, et al. Drosophila endogenous small RNAs bind to Argonaute 2 in somatic cells. Nature. 2008;453(7196):793-7.

29. Couvillion MT, Sachidanandam R, Collins K. A growth-essential Tetrahymena Piwi protein carries tRNA fragment cargo. Gene Dev. 2010:24(24):2742-7.

30. Burroughs AM, Ando Y, de Hoon MJ, Tomaru Y, Suzuki H, Hayashizaki $Y$, et al. Deep-sequencing of human Argonaute-associated small RNAs provides insight into miRNA sorting and reveals Argonaute association with RNA fragments of diverse origin. RNA Biol. 2011;8(1):158-77.

31. Pekarsky Y, Balatti V, Palamarchuk A, Rizzotto L, Veneziano D, Nigita G, et al. Dysregulation of a family of short noncoding RNAs, tsRNAs, in human cancer. Proc. Natl. Acad. Sci. 2016:113(18):5071-6.

32. Thompson DM, Parker R. Stressing out over tRNA cleavage. Cell. 2009;138(2):215-9.

33. Sun C, Fu Z, Wang S, Li J, Li Y, Zhang Y, et al. Roles of tRNA-derived fragments in human cancers. Cancer Lett. 2018:414:16-25.

34. Veneziano D, Tomasello L, Balatti V, Palamarchuk A, Rassenti LZ, Kipps $\mathrm{TJ}$, et al. Dysregulation of different classes of tRNA fragments in chronic lymphocytic leukemia. Proc. Natl. Acad. Sci. 2019;116(48):24252-8.

35. Chen Q, Yan M, Cao Z, Li X, Zhang Y, Shi J, et al. Sperm tsRNAs contribute to intergenerational inheritance of an acquired metabolic disorder. Science. 2016:351(6271):397 
36. Levitz R, Chapman D, Amitsur M, Green R, Snyder L, Kaufmann GJEJ. The optional E. coli prr locus encodes a latent form of phage T4-induced anticodon nuclease. EMBO J. 1990;9(5):1383-9.

37. Singh NK, Paz E, Kutsher Y, Reuveni M, Lers A. Tomato T2 ribonuclease LE is involved in the response to pathogens. Mol. Plant Pathol. 2020;7:895-906.

38. Ren B, Wang X, Duan J, Ma J. Rhizobial tRNA-derived small RNAs are signal molecules regulating plant nodulation. Science. 2019;365(6456):919.

39. Alves CS, Vicentini R, Duarte GT, Pinoti VF, Vincentz M, Nogueira FT. Genome-wide identification and characterization of tRNA-derived RNA fragments in land plants. Plant Mol. Biol. 2017;93(1-2):35-48.

40. Wang Y, Li H, Sun Q, Yao Y. Characterization of small RNAs derived from tRNAs, rRNAs and snoRNAs and their response to heat stress in wheat deedlings. PLoS One. 2016;11(3):e0150933.

41. Gupta N, Singh A, Zahra S, Kumar S. PtRFdb: a database for plant transfer RNA-derived fragments. Database (Oxford) 2018: bay063.

42. Choulet F, Wicker T, Rustenholz C, Paux E, Salse J, Leroy P, et al. Megabase level sequencing reveals contrasted organization and evolution patterns of the wheat gene and transposable element spaces. Plant Cell. 2010;22(6):1686-701.

43. Ma HX, Bai GH, Zhang X, Lu WZ. Main effects, epistasis, and environmental interactions of quantitative trait Loci for fusarium head blight resistance in a recombinant inbred population. Phytopathology. 2006:96(5):534-41.

44. Schweiger W, Steiner B, Ametz C, Siegwart G, Wiesenberger G, Berthiller $F$, et al. Transcriptomic characterization of two major Fusarium resistance quantitative trait loci (QTLs), Fhb1 and Qfhs.ifa-5A, identifies novel candidate genes. Mol. Plant Pathol. 2013;14(8):772-85.

45. Suzuki T, Sato M, Takeuchi T. Evaluation of the effects of five QTL regions on Fusarium head blight resistance and agronomic traits in spring wheat (Triticum aestivum L.). Breed Sci. 2012:62(1):11-7.

46. Zhou MP, Hayden MJ, Zhang ZY, Lu WZ, Ma HX. Saturation and mapping of a major Fusarium head blight resistance QTL on chromosome 3BS of Sumai 3 wheat. J. Genet. 2010;51(1):19-25.

47. Bai G, Kolb FL, Shaner G, Domier LL. Amplified fragment length polymorphism markers linked to a major quantitative trait locus controlling scab resistance in wheat. Phytopathology. 1999;89(4):343-8.

48. Ge L, Zhang N, Li D, Wu Y, Wang H, Wang J. Circulating exosomal small RNAs are promising non-invasive diagnostic biomarkers for gastric cancer. J. Cell Mol. Med. 2020;24:14502-13.

49. Langmead B, Trapnell C, Pop M, Salzberg SL. Ultrafast and memoryefficient alignment of short DNA sequences to the human genome. Genome Biol. 2009;10(3):R25.

50. Li R, Li Y, Kristiansen K, Wang J. SOAP: short oligonucleotide alignment program. Bioinformatics. 2008;24(5):713-4.

51. Kim D, Langmead B, Salzberg SL. HISAT: a fast spliced aligner with low memory requirements. Nat. Methods. 2015;12(4):357-60.

52. Li B, Dewey CN. RSEM: accurate transcript quantification from RNASeq data with or without a reference genome. BMC Bioinformatics. 2011;12(1):323.

53. Love MI, Huber W, Anders S. Moderated estimation of fold change and dispersion for RNA-seq data with DESeq2. Genome Biol. 2014;15(12):550.

54. Abdi H. The Bonferonni and Šidák Corrections for Multiple Comparisons. Encyclopedia of Measurement and Statistics. 2007;1:1-9.

55. Varkonyi-Gasic E, Wu R, Wood M, Walton EF, Hellens RP. Protocol: a highly sensitive RT-PCR method for detection and quantification of microRNAs. Plant Methods. 2007;3:12.

56. Chen C, Chen H, Zhang Y, Thomas HR, Frank MH, He Y, et al. TBtools: an integrative toolkit developed for interactive analyses of big biological data. Mol. Plant. 2020;13(8):1194-202.

57. Li H. Complexes of tRNA and maturation enzymes: shaping up for translation. Curr. Opin. Struct. Biol. 2007;17(3):293-301.

58. Phizicky EM, Hopper AK. tRNA biology charges to the front. Genes Dev. 2010;24(17):1832-60.

59. Hillwig MS, Contento AL, Meyer A, Ebany D, Bassham DC, Maclntosh GC. RNS2, a conserved member of the RNase T2 family, is necessary for ribosomal RNA decay in plants. Proc. Natl. Acad. Sci. 2011;108(3):1093-8.

60. Lee YS, Shibata Y, Malhotra A, Dutta A. A novel class of small RNAs: tRNAderived RNA fragments (tRFs). Genes Dev. 2009;23(22):2639-49.

61. Maraia RJ, Lamichhane TN. 3' processing of eukaryotic precursor tRNAs. Wiley Interdiscip Rev. RNA. 2011;2(3):362-75.
62. Haussecker D, Huang Y, Lau A, Parameswaran P, Fire AZ, Kay MA. Human tRNA-derived small RNAs in the global regulation of RNA silencing. RNA 2010;16(4):673-95.

63. Hollenstein K, Frei DC, Locher KP. Structure of an ABC transporter in complex with its binding protein. Nature. 2007:446(7132):213-6.

64. Boyes DC, Nam J, Dangl JL. The Arabidopsis thaliana RPM1 disease resistance gene product is a peripheral plasma membrane protein that is degraded coincident with the hypersensitive response. Proc. Natl. Acad. Sci. 1998;95(26):15849.

65. Bittner-Eddy PD, Crute IR, Holub EB, Beynon JL. RPP13 is a simple locus in Arabidopsis thaliana for alleles that specify downy mildew resistance to different avirulence determinants in Peronospora parasitica. Plant J. 2000;21(2):177-88.

66. Kunkel BN, Bent AF, Dahlbeck D, Innes RW, Staskawicz BJ. RPS2, an Arabidopsis disease resistance locus specifying recognition of Pseudomonas syringae strains expressing the avirulence gene avrRpt2. The Plant Cell. 1993;5(8):865-75.

67. Liu X, Liu H, He J, Zhang S, Han H, Wang Z, et al. RIN13-mediated disease resistance depends on the SNC1-EDS1/PAD4 signaling pathway in Arabidopsis. J. Exp. Bot. 2020;71(22):7393-404.

68. Aslam M, Ullah A, Paramasivam N, Kandasamy N, Naureen S, Badshah M, et al. Segregation and potential functional impact of a rare stop-gain PABPC4L variant in familial atypical Parkinsonism. Sci. Rep. 2019;9(1):13576.

69. Caro E, Stroud H, Greenberg MV, Bernatavichute YV, Feng S, Groth M, et al. The SET-domain protein SUVR5 mediates H3K9me2 deposition and silencing at stimulus response genes in a DNA methylation-independent manner. PLoS Genet. 2012;8(10):e1002995.

70. Lagresle-Peyrou C, Six EM, Picard C, Rieux-Laucat F, Michel V, Ditadi A, et al. Human adenylate kinase 2 deficiency causes a profound hematopoietic defect associated with sensorineural deafness. Nat. Genet. 2009;41(1):106-11.

\section{Publisher's Note}

Springer Nature remains neutral with regard to jurisdictional claims in published maps and institutional affiliations.

Ready to submit your research? Choose BMC and benefit from

- fast, convenient online submission

- thorough peer review by experienced researchers in your field

- rapid publication on acceptance

- support for research data, including large and complex data types

- gold Open Access which fosters wider collaboration and increased citations

- maximum visibility for your research: over $100 \mathrm{M}$ website views per year

At BMC, research is always in progress.

Learn more biomedcentral.com/submissions 